\title{
On the Spectrum of Curved Planar Waveguides
}

\author{
By \\ David KREJČIŘíK* and Jan KŘÍŽz**
}

\begin{abstract}
The spectrum of the Laplace operator in a curved strip of constant width built along an infinite plane curve, subject to three different types of boundary conditions (Dirichlet, Neumann and a combination of these ones, respectively), is investigated. We prove that the essential spectrum as a set is stable under any curvature of the reference curve which vanishes at infinity and find various sufficient conditions which guarantee the existence of geometrically induced discrete spectrum. Furthermore, we derive a lower bound to the distance between the essential spectrum and the spectral threshold for locally curved strips. The paper is also intended as an overview of some new and old results on spectral properties of curved quantum waveguides.
\end{abstract}

\section{Contents}

$\S 1 . \quad$ Introduction

$\S 2 . \quad$ Scope of the Paper

$\S 3 . \quad$ Preliminaries

$\S 3.1 . \quad$ Configuration space

$\S 3.2$. The Laplacian

$\S 3.3$. Straight strips

$\S 4$. Main Results

Communicated by H. Okamoto. Received June 7, 2004. Revised August 20, 2004.

2000 Mathematics Subject Classification(s): 58J50, 35P15, 35Q40, 47A75, 49R50, 81Q10.

*Departamento de Matemática, Instituto Superior Técnico, Av. Rovisco Pais, 1049-001 Lisboa, Portugal.

e-mail: dkrej@math.ist.utl.pt

Also on leave of absence from Department of Theoretical Physics, Nuclear Physics Institute, Academy of Sciences, 25068 Rež near Prague, Czech Republic

** Department of Physics, Faculty od Education, University of Hradec Králové, Rokitanského 62, 50003 Hradec Králové, Czech Republic.

e-mail: jan.kriz@uhk.cz

(C) 2005 Research Institute for Mathematical Sciences, Kyoto University. All rights reserved. 
$\S 5$. Essential Spectrum

$\S 6$. Curvature-induced Spectrum

$\S 6.1$. The existence

$\S 6.2$. The estimates on the spectral threshold

$\S 7 . \quad$ Conclusions

Acknowledgements

References

\section{$\S 1$. Introduction}

Let $\Omega$ be a region (i.e. open connected set) in $\mathbb{R}^{n}, n \geq 1$, with sufficiently regular boundary $\partial \Omega$, and consider the corresponding Laplacian $-\Delta$ on $L^{2}(\Omega)$ with mixed Dirichlet-Neumann boundary conditions. If $\Omega$ is bounded, then it is well known that the spectrum of the Laplacian is purely discrete, and properties of the eigenvalues have been intensively studied. On the other hand, it is easy to see that the spectrum is $[0, \infty)$, i.e. purely essential, if $\Omega$ is unbounded and sufficiently extended at infinity (namely, it contains arbitrarily large balls). Although it was shown already by F. Rellich in 1948 [71] that there exist unbounded regions whose spectrum contains discrete eigenvalues (or it is even purely discrete!), the spectral theory for the eigenvalues has attracted much less attention than in the bounded case.

However, recent advent of mesoscopic physics has given a fresh impetus to study the (discrete) spectrum of the Laplacian in unbounded regions. For, let us recall that the quantum Hamiltonian $H$ of a free spin-less particle of effective mass $m^{*}$ constrained to a spatial region $\Omega$, i.e. $H=-\hbar^{2} /\left(2 m^{*}\right) \Delta$ on $L^{2}(\Omega)$, represents a reasonable mathematical model for the dynamics in various semiconductor structures devised and produced in the laboratory nowadays. Here it is mostly natural to consider the Dirichlet boundary conditions on $\partial \Omega$ corresponding to a large chemical potential barrier, however, other situations modelling the impenetrable walls of $\Omega$ (in the sense that there is no probability current through the boundary) may be relevant as well (see e.g. $[62,63]$ ) and can in principle model different types of interphase in a solid. We refer to $[18,65,53]$ for the physical background and references. An important category of these systems is represented by so-called quantum waveguides which are modelled by infinitely stretched tubular regions in $\mathbb{R}^{n}$ with $n=2,3$.

The simplest situation occurs if $\Omega$ is an infinite plane strip, i.e. a tubular neighbourhood of constant width along an infinite curve in $\mathbb{R}^{2}$. In 1989, 
P. Exner and P. Šeba [42] demonstrated the existence of discrete spectrum for the Dirichlet Laplacian in curved strips which were asymptotically straight and sufficiently thin. Numerous subsequent studies improved their result and generalized it to space tubes $[52,72,18]$. For more information and other spectral and scattering properties, see the review paper [18] and references therein. An important improvement was made by J. Goldstone and R. L. Jaffe in 1992 [52]; the authors introduced a variational argument which enables them to demonstrate the existence of discrete eigenvalues without the restriction on the width of the strip. The paper [58] deals with a more general situation where the strip is not constructed in $\mathbb{R}^{2}$ but in a two-dimensional Riemannian manifold. The evidently more complicated case of layers, i.e. $\Omega$ is a tubular neighbourhood about a complete non-compact surface in $\mathbb{R}^{3}$, was investigated in $[19,20,38,12,64]$.

A common property of the Dirichlet systems cited above is that a bending of a straight strip or layer generates discrete eigenvalues below the essential spectrum, i.e. geometrically induced quantum bound states, which are known to disturb the particle transport. The result is also interesting from the semiclassical point of view because there are no classical closed trajectories in the tubes in question, apart from a zero measure set of initial conditions in the phase space. Hence, this is a pure quantum effect of geometrical origin.

On the mathematical side, the results are of interest because the tubular neighbourhoods represent a class of so-called quasi-cylindrical regions, for which the existence of discrete spectrum is a non-trivial property. We refer to the books [51] and [21] for a classification of Euclidean regions and basic properties of the spectrum of the Dirichlet Laplacian as related to the form of an unbounded region.

The spectral results become richer if one considers a combination of Dirichlet and Neumann boundary conditions $[16,17]$. Here the problem is interesting even for straight strips and much less studied in the literature.

Apart from the curved quantum waveguides, the discrete spectrum can be also generated by a local deformation of the boundary $\partial \Omega$ of straight tubes and layers $[9,8,47]$, via introducing an obstacle $[23,14,2]$ or impurities modelled by a Dirac interaction $[26,37,39,40]$, coupling several waveguides by a window $[44,45,46,6]$, etc. The spectrum of periodically and randomly curved waveguides was investigated in [76, 74] and [55], respectively. Finally, let us mention systems where $\Omega=\mathbb{R}^{n}, n=2,3$, and the quantum waveguide is introduced by means of a magnetic field $[28,36,29]$ or a strong Dirac interaction supported by an infinite curve or surface [27, 48, 30, 31, 24, 32, 33, 34, 35, 41]. 
The present paper is devoted to a study of the interplay between the geometry, boundary conditions (we consider uniform Dirichlet, Neumann or a combination of these ones) and the spectral properties of the Laplacian in the infinite planar curved strips. We referred above to the theory of quantum waveguides as our main physical motivation. Let us conclude this section by mentioning other fields in physics where this might be a reasonable mathematical model.

Considerable current interest in designing integrated optoelectronic circuits involves (classical) electromagnetic waveguides as a set of essential components. In two-dimensional structures, planar symmetry implies that the waveguide modes can have transversally electric, respectively transversally magnetic polarizations corresponding to Dirichlet, respectively Neumann boundary conditions, $c f$ [66]. Maxwell's equations yield the similar spectral problem as above, the difference is only in the physical meaning of the spectral parameter.

The eigenvalues of the Neumann Laplacian may be also regarded as velocity potentials of an inviscid, irrotational fluid or trapped vibrational modes of an acoustic waveguide. We refer to $[23,14]$ for the considerable applied literature on such problems.

Combinations of Dirichlet and Neumann boundary conditions appear as a natural generalization of the uniform boundary conditions. A physical system satisfying such a combination is the Earth-ionosphere waveguide: it is known that for very low frequencies the electromagnetic wave dynamics between the Earth and the ionosphere can be approximated as a propagation between the plates with the perfect electric (the Earth) and perfect magnetic (the ionosphere) conductors, see [68] and references therein. Problems of this type arises naturally in many other areas of physics, most notably in theoretical studies of superconductivity, photonic crystals, etc.

\section{$\S 2 . \quad$ Scope of the Paper}

The main aim of the present paper is to study the geometrically induced (discrete) spectrum of the operator $H^{\iota}$ defined as the Laplacian $-\Delta$ on $L^{2}(\Omega)$, where $\Omega$ is a (one-sided) tubular neighbourhood of a fixed width $d>0$ along an infinite plane curve $\Gamma$ of curvature $k$, see Figure 1 . We adopt suitable hypotheses ( $c f\langle\mathbf{H}\rangle$ below) in order to ensure that the boundary $\partial \Omega$ consists of two parallel connected embedded curves of class $C^{2}$. The index $\iota$ will distinguish three different types of boundary conditions considered here. Namely, we consider the recently widely investigated Dirichlet boundary condition $(\iota:=D)$, the Neumann boundary condition $(\iota:=N)$ and the simplest combination of the both just mentioned $(\iota:=D N)$ : the Dirichlet boundary condition imposed 


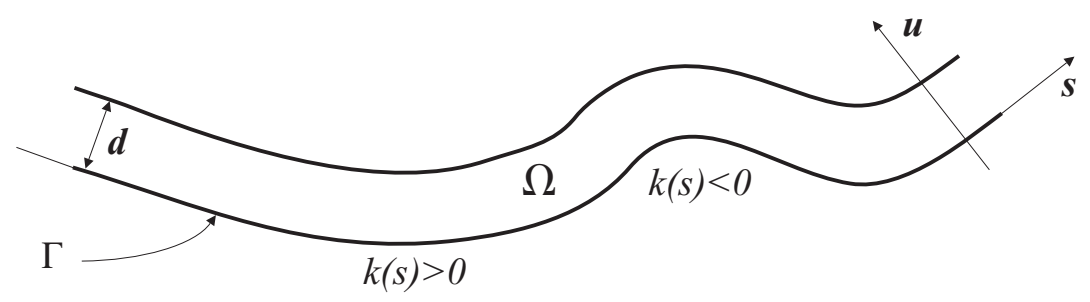

Figure 1. Configuration space $\Omega$ defined as a strip over an infinite curve $\Gamma$ in $\mathbb{R}^{2}$.

on one connected component of $\partial \Omega$ and the Neumann condition on the other one.

If the reference curve $\Gamma$ is a straight line, then it is rather a textbook exercise to analyse the operator $H^{\iota}$ by means of a separation of variables and conclude that its spectrum is purely absolutely continuous and equals the interval $\left[E_{1}^{\iota}, \infty\right)$, where the non-negative value $E_{1}^{\iota}$ is determined by the respective boundary conditions, $c f(3.10)$. However, the spectral problem for $H^{\iota}$ becomes always difficult whenever $\Gamma$ is curved, and two basic questions arise in this context:

1. Which geometry preserves the essential spectrum $\left[E_{1}^{\iota}, \infty\right)$ ?

2. Which geometry produces a spectrum below $E_{1}^{\iota}$ ?

These questions represent ultimate concern of this paper. We try to make a survey of known answers and contribute to the problem by our own results. Furthermore, if the spectrum below $E_{1}^{\iota}$ exists, we establish various estimates of the spectral threshold inf $\sigma\left(H^{\iota}\right)$. It should be stressed here that the existence of discrete spectrum, i.e. the issue mentioned in Introduction, is proved whenever the considered geometry is in accordance with both the above questions (because then the spectrum below $E_{1}^{\iota}$ consists of isolated eigenvalues of finite multiplicity only).

Concerning the first question, we show that the essential spectrum of a curved strip coincides with the spectrum of the straight one provided the reference curve $\Gamma$ is straight asymptotically in the sense that its curvature vanishes at infinity, $c f$ Theorem 4.1. Although this sufficient condition is very natural and in perfect accordance with the intuition, it is for the first time in this paper when the essential spectrum is localized without imposing any additional conditions (e.g., about the decay of the derivatives of curvature at infinity, 
cf $[18,72,17])$. The progress has become possible due to a general characterization of essential spectrum adopted from a paper by Y. Dermenjian et al. [15] ( $c f$ our Lemma 5.1), which is for our purposes more suitable than the classical Weyl criterion. On the other hand, periodic strips are discussed as an illustration of asymptotically non-straight geometry which does change the essential spectrum.

The answer to the second question depends substantially on the choice of boundary conditions. First of all, notice that the question does not make sense for the Neumann strips because $E_{1}^{N}=0$, cf Theorem 4.2. A characteristic property of the Dirichlet strips is that any bending of the reference curve $\Gamma$ pushes the infimum of the spectrum below the spectral threshold $E_{1}^{D}>0$ of the corresponding straight strip, $c f$ Theorem 4.3. This property was shown first in [42] for sufficiently thin strips and the proof for more general cases was introduced in [52]. On the other hand, the case of combined Dirichlet-Neumann boundary conditions was introduced quite recently in [17]. The authors established the existence of spectrum below $E_{1}^{D N}>0$ provided the total bending angle of $\Gamma$ (i.e. the integral of curvature, $c f(4.1)$ ) has a suitable sign. In this paper, we generalize this result and add two new sufficient conditions, cf Theorem 4.4. We also derive an interesting result on the number of discrete eigenvalues of $H^{D N}$, cf Proposition 4.1

Finally, when $\Gamma$ is curved only locally, we derive an upper bound to the spectral threshold, i.e. $\inf \sigma\left(H^{\iota}\right)$, for the Dirichlet strip and the one with combined Dirichlet-Neumann boundary condition, $c f$ Theorem 4.5. In particular, we find important qualitative differences between these two respective results. Making the curvature small, the leading term in the estimate of the difference $\inf \sigma\left(H^{D}\right)-E_{1}^{D}$ is proportional to the fourth power of the total bending angle, while it is the second power what one obtains for the Dirichlet-Neumann case, cf Remark 6.3. Another interesting difference appears when we are shrinking the width of the strip to zero, $c f$ Remark 6.4. These estimates are new in the theory of curved quantum waveguides. We can only compare them with the eigenvalue asymptotics for mildly curved, respectively thin, Dirichlet strips established in [18]. Let us note that a similar estimate for straight, windowcoupled waveguides was given in [45, 46], see also [7].

All our proofs of the statements concerning the existence and properties of the spectrum below $E_{1}^{\iota}$ are based on a variational strategy. The corner stone of them, i.e. the construction of a suitable trial function, follows the idea of [52], see also $[18,72]$. 
The paper is organized as follows. Section 3 is devoted to some preliminary material in order to be able to state precisely the main results of the paper, i.e. Theorems 4.1-4.5, in the subsequent Section 4. The proofs and discussions of the Theorems are presented in Sections 5 and 6 . We conclude the paper by Section 7 , where some open problems and directions of a future research are mentioned.

\section{§3. Preliminaries}

\section{§3.1. Configuration space}

Let $\Gamma$ be a unit-speed infinite plane curve, i.e. the (image of the) $C^{2}$ smooth embedding $\Gamma: \mathbb{R} \rightarrow \mathbb{R}^{2}:\left\{s \mapsto\left(\Gamma^{1}(s), \Gamma^{2}(s)\right)\right\}$ satisfying $|\dot{\Gamma}(s)|=1$ for all $s \in \mathbb{R}$ (the arc-length parameter of the curve). The function $N:=\left(-\dot{\Gamma}^{2}, \dot{\Gamma}^{1}\right)$ defines a unit normal vector field and the couple $(\dot{\Gamma}, N)$ gives a distinguished Frenet frame, cf [56, Chap. 1]. The curvature is defined through the FrenetSerret formulae by $k:=\operatorname{det}(\dot{\Gamma}, \ddot{\Gamma})$. We note that $k$ is a continuous function of the arc-length parameter and the sign of $k(s)$ is defined uniquely up to the re-parameterization $s \mapsto-s$. It is also worth to notice that the curve $\Gamma$ is fully determined (except for its position and orientation in the plane) by the curvature function $k$ only, cf [60, Sec. II. 20].

Let $d>0, I:=(0, d)$ and $\Omega_{0}:=\mathbb{R} \times I$ be a straight strip of width $d$. We define a curved strip of the same width based on $\Gamma$ via $\Omega:=\mathcal{L}\left(\Omega_{0}\right)$, where

$$
\mathcal{L}: \mathbb{R}^{2} \rightarrow \mathbb{R}^{2}:\{(s, u) \mapsto \Gamma(s)+u N(s)\} .
$$

Through all the paper, we always assume that

$\langle\mathrm{H}\rangle \quad \Omega$ is not self-intersecting and $k \in L^{\infty}(\mathbb{R})$ with $d\left\|k_{+}\right\|_{\infty}<1$,

where $k_{ \pm}:=\max \{0, \pm k\}$. Then $s \mapsto \mathcal{L}(s, u)$ for $u \in \bar{I}$ fixed traces out a parallel curve at a distance $|u|$ from $\Gamma$ and $u \mapsto \mathcal{L}(s, u)$ for $s \in \mathbb{R}$ fixed is a straight line orthogonal to $\Gamma$ at $s$. Furthermore, the mapping $\mathcal{L}: \Omega_{0} \rightarrow \Omega$ is a $C^{1}$-diffeomorphism and its inverse determines a system of natural "coordinates" $(s, u)$ in a neighbourhood of $\Gamma$. We remark that under our assumption $\langle\mathrm{H}\rangle$ the curve $\mathcal{L}(\mathbb{R} \times\{u\})$ is of class $C^{2}$ for any fixed $u \in \bar{I}$, in particular, this claim holds true for both the boundary curves.

Remark 3.1. In this paper, we adopt the standard component notation of tensor analysis together with the repeated indices convention. The range of indices is 1,2 and they are associated with the above mentioned coordinates via $(1,2) \leftrightarrow(s, u)$. The partial derivatives are marked by a comma with the index. 
By virtue of the Frenet-Serret formulae, the metric tensor of $\Omega$ in these coordinates, i.e. $G_{i j}:=\mathcal{L}_{, i} \cdot \mathcal{L}_{, j}$ where "." denotes the scalar product in $\mathbb{R}^{2}$, has the following diagonal form

$$
\left(G_{i j}(s, u)\right)=\left(\begin{array}{cc}
(1-u k(s))^{2} & 0 \\
0 & 1
\end{array}\right) .
$$

Its determinant, $G:=\operatorname{det}\left(G_{i j}\right)$, defines through $d \Omega:=G(s, u)^{\frac{1}{2}} d s d u$ the area element of the strip. By virtue of the second part of the assumption $\langle\mathbf{H}\rangle$, it is clear that the metric (3.2) is uniformly elliptic. In particular, we have the following useful estimates:

$$
\forall(s, u) \in \Omega_{0}: \quad C_{-} \leq 1-u k(s) \leq C_{+} \quad \text { with } \quad C_{ \pm}:=1 \pm d\left\|k_{\mp}\right\|_{\infty} .
$$

\section{§3.2. The Laplacian}

Our object of interest is the Laplacian $-\Delta$ on $L^{2}(\Omega)$, subject to various boundary conditions imposed on $\partial \Omega$. Our basic strategy is to use the diffeomorphism $\mathcal{L}: \Omega_{0} \rightarrow \Omega$ in order to replace the simple operator $-\Delta$ on the complicated Hilbert space $L^{2}(\Omega)$ by a more complicated operator $H^{\iota}$ on the simpler Hilbert space $\mathcal{H}:=L^{2}\left(\Omega_{0}, d \Omega\right)$. In particular, $H^{D}$ is the operator replacing the Laplacian with Dirichlet boundary condition, $H^{N}$ corresponds to the Neumann boundary condition and $H^{D N}$ has the Dirichlet boundary condition imposed on the reference curve $\Gamma \equiv \mathcal{L}(\mathbb{R} \times\{0\})$ and the Neumann one imposed on the opposite boundary $\mathcal{L}(\mathbb{R} \times\{d\})$. Sometimes, we shall use the common superscript $\iota \in\{D, N, D N\}$ to consider two or all of the three different situations simultaneously.

More precisely, the operators $H^{\iota}$ are introduced as the unique self-adjoint operators associated on $\mathcal{H}$ with the quadratic forms $Q^{\iota}$ defined by

$$
\begin{aligned}
Q^{\iota}[\psi] & :=\left(\psi_{, i}, G^{i j} \psi_{, j}\right), \\
\operatorname{Dom} Q^{D} & :=W_{0}^{1,2}\left(\Omega_{0}, d \Omega\right), \\
\operatorname{Dom} Q^{N} & :=W^{1,2}\left(\Omega_{0}, d \Omega\right), \\
\operatorname{Dom} Q^{D N} & :=\left\{\psi \in W^{1,2}\left(\Omega_{0}, d \Omega\right) \mid \psi(s, 0)=0 \quad \text { for a.e. } s \in \mathbb{R}\right\} .
\end{aligned}
$$

Here and in what follows, $\left(G^{i j}\right)$ stands for the inverse of $\left(G_{i j}\right),(\cdot, \cdot)$ and $\|\cdot\|$ denotes the scalar product and the norm in $\mathcal{H}$, respectively, and $\psi(\cdot, 0)$ means the trace of the function $\psi$ on the boundary part $\mathcal{L}(\mathbb{R} \times\{0\})$.

Remark 3.2. Since the metric $\left(G_{i j}\right)$ is uniformly elliptic due to $(3.3)$, it is not necessary to take into account the measure $d \Omega$ in (3.5), (3.6) and (3.7). 
Remark 3.3 (Operators associated with $Q^{\iota}$ ). We have

$$
H^{\iota}=-G^{-\frac{1}{2}} \partial_{i} G^{\frac{1}{2}} G^{i j} \partial_{j},
$$

which is a general expression for the Laplacian in a manifold equipped with a metric $\left(G_{i j}\right)$. The equality in (3.8) must be understood in the form sense if the curvature $k$ is not differentiable (which is the case we are particularly concerned to deal with in this paper). Nevertheless, assuming that the reference curve $\Gamma$ is, say, $C^{3}$-smooth, then the metric is differentiable and, putting (3.2) into (3.8), we can write

$$
H^{\iota}=-\frac{1}{(1-u k(s))^{2}} \partial_{s}^{2}-\frac{u \dot{k}(s)}{(1-u k(s))^{3}} \partial_{s}-\partial_{u}^{2}+\frac{k(s)}{1-u k(s)} \partial_{u}
$$

as an operator identity on the functions from Dom $H^{\iota}$. Moreover, the operator domain Dom $H^{\iota}$ is exactly that subset of the space $W^{2,2}\left(\Omega_{0}\right)$ whose elements satisfy the corresponding boundary conditions on $\partial \Omega_{0}$ in the classical sense, cf [61].

\section{§3.3. Straight strips}

If the strip is straight in the sense that $k \equiv 0$, i.e. $k$ is equal to zero everywhere on $\mathbb{R}$, then the Laplacian coincides with the decoupled operator

$$
H_{0}^{\iota}:=\overline{-\Delta^{\mathbb{R}} \otimes I d+I d \otimes\left(-\Delta_{\iota}^{I}\right)} \quad \text { on } \quad L^{2}(\mathbb{R}) \otimes L^{2}(I),
$$

where $I d$ denotes the identity operator on appropriate spaces. The operators on the transverse section, $-\Delta_{\iota}^{I}$, are the usual Laplacians on $L^{2}(I)$ with the Dirichlet boundary conditions if $\iota=D$, the Neumann conditions if $\iota=N$, or the Dirichlet condition at 0 and the Neumann one at $d$ if $\iota=D N$. The eigenvalues of $-\Delta_{\iota}^{I}$ are given by

$$
E_{n}^{D}:=(\pi / d)^{2} n^{2}, \quad E_{n}^{N}:=(\pi / d)^{2}(n-1)^{2}, \quad E_{n}^{D N}:=(\pi / d)^{2}\left(n-\frac{1}{2}\right)^{2},
$$

where $n \in \mathbb{N} \backslash\{0\}$. The corresponding family of normalized eigenfunctions $\left\{\chi_{n}^{\iota}\right\}_{n=1}^{\infty}$ can be chosen in the following way:

$$
\begin{aligned}
& \chi_{n}^{\iota}(u):=\sqrt{\frac{2}{d}} \sin \sqrt{E_{n}^{\iota}} u \text { for } \quad \iota \in\{D, D N\} ; \\
& \chi_{n}^{N}(u):=\left\{\begin{array}{lll}
\sqrt{\frac{1}{d}} & \text { if } & n=1, \\
\sqrt{\frac{2}{d}} \cos \sqrt{E_{n}^{N}} u & \text { if } & n \geq 2 .
\end{array}\right.
\end{aligned}
$$


In view of (3.9) and [69, Thm. VIII.33], the straight strip has an absolutely continuous spectrum starting from the first eigenvalue of the transverse Laplacian, i.e.,

$$
\sigma\left(H_{0}^{\iota}\right)=\sigma_{\mathrm{ess}}\left(H_{0}^{\iota}\right)=\left[E_{1}^{\iota}, \infty\right) .
$$

\section{§4. Main Results}

As we have seen, the essential spectrum of a straight strip, i.e. $k \equiv 0$, is the interval $\left[E_{1}^{\iota}, \infty\right)$. In Section 5 , we prove that the same spectral result holds for any curved strip which is straight asymptotically in the sense that the curvature $k$ vanishes at infinity, i.e.,

$\langle d\rangle \quad k(s) \underset{|s| \rightarrow \infty}{\longrightarrow} 0$.

Theorem 4.1 (Essential spectrum). $\quad$ Suppose $\langle\mathrm{H}\rangle$. If the strip satisfies $\langle\mathrm{d}\rangle$, then

$$
\sigma_{\mathrm{ess}}\left(H^{\iota}\right)=\left[E_{1}^{\iota}, \infty\right) \quad \text { for } \quad \iota \in\{D, N, D N\} .
$$

To the best of our knowledge, the spectrum of the Neumann Laplacian $H^{N}$ has been previously investigated just for strips which were straight and contained an obstacle, [23, 14]. Hence, our Theorem 4.1 represents a quite new result concerning the spectral theory of curved Neumann strips.

The Dirichlet-Neumann case, i.e. $\iota=D N$, was previously considered just in the recent letter [17]. It is mentioned there that $\inf \sigma_{\text {ess }}\left(H^{D N}\right)=E_{1}^{D N}$ provided $k$ has a compact support. Here we have proved that the whole interval $\left[E_{1}^{D N}, \infty\right)$ is in the essential spectrum under much weaker condition $\langle\mathrm{d}\rangle$.

Although the case of Dirichlet strips, i.e. $\iota=D$, has already been considered in many works, our Theorem 4.1 represents a new result in this situation as well, since it is for the first time when the whole essential spectrum has been localized under a condition which does not contain derivatives of $k$. Some decay assumptions about the derivatives of the curvature were even required in order to localize the threshold $\inf \sigma_{\text {ess }}\left(H^{D}\right)$ itself in the previous works, cf $[18,72]$. (An exception is the paper [58] where, however, only a lower bound on the threshold is given.) Let us mention that the result of Theorem 4.1 was achieved in the thesis [57] under an additional condition about vanishing of the first derivative of $k$.

Since $H^{N}$ is non-negative, it follows immediately from Theorem 4.1 that there is no discrete spectrum in asymptotically straight Neumann strips. 
Theorem 4.2 (Neumann case). Suppose $\langle\mathrm{H}\rangle$. Then

$$
\inf \sigma\left(H^{N}\right)=E_{1}^{N} \equiv 0
$$

Consequently, if the strip is asymptotically straight, i.e. $\langle\mathrm{d}\rangle$, then

$$
\sigma\left(H^{N}\right)=\sigma_{\mathrm{ess}}\left(H^{N}\right)=[0, \infty),
$$

i.e., $\sigma_{\text {disc }}\left(H^{N}\right)=\emptyset$.

Here the fact that the spectral threshold of $H^{N}$ starts exactly at 0 for any strip can be easily proved by means of a suitable trial function ( $c f$ Proposition 6.1).

An interesting result in the theory of quantum waveguides is that the curved geometry may produce a non-trivial spectrum below the energy $E_{1}^{\iota}$ for $\iota \in\{D, D N\}$. The phenomenon is examined in this paper. Notice that any result of the type inf $\sigma\left(H^{\iota}\right)<E_{1}^{\iota}$ together with the decay condition $\langle$ d $\rangle$ yield that the spectrum below $E_{1}^{\iota}$ consists of isolated eigenvalues of finite multiplicity only, i.e. $\sigma_{\mathrm{disc}}\left(H^{\iota}\right) \neq \emptyset$. However, we do not restrict ourselves to the particular case of asymptotically straight strips, i.e., the geometrically induced spectrum below $E_{1}^{\iota}$ may have a non-zero Lebesgue measure, too.

Sufficient conditions for the Laplacians $H^{\iota}$ with $\iota \in\{D, D N\}$ to have a non-empty spectrum below $E_{1}^{\iota}$ are known. In particular, any non-trivial curvature of the reference curve pushes the spectrum of $H^{D}$ down the corresponding spectral threshold of the straight strip.

Theorem 4.3 (Dirichlet case). Suppose $\langle\mathrm{H}\rangle$.

If $k \neq 0$, then $\inf \sigma\left(H^{D}\right)<E_{1}^{D}$.

Consequently, if the strip is not straight but it is straight asymptotically, i.e. $\langle\mathrm{d}\rangle$, then $H^{D}$ has at least one eigenvalue of finite multiplicity below its essential spectrum $\left[E_{1}^{D}, \infty\right)$, i.e., $\sigma_{\text {disc }}\left(H^{D}\right) \neq \emptyset$.

This property was shown first in [42] for sufficiently thin strips with a rapidly decaying curvature and since various improvements have been achieved (see the references mentioned in Introduction, mainly [52]). We find useful to make a proof of Theorem 4.3 in Section 6.1 since it can be made simultaneously with the proof of the new result contained in condition (a) of Theorem 4.4 below.

As for the operator $H^{D N}$, its spectrum was studied for the first time in the recent letter [17]. It shows that the position of the infimum of spectrum 
essentially depends on the sign of the total bending angle

$$
\alpha:=\int_{\mathbb{R}} k(s) d s,
$$

which is well defined if we assume that the curvature is integrable. In detail, the authors of [17] proved that: i) the spectrum of $H^{D N}$ in a non-trivially curved strip starts below $E_{1}^{D N}$ provided $\alpha \leq 0$ and the curvature $k$ is nonpositive out of some bounded interval. On the other hand, ii) if $k(s) \geq 0$ for all $s \in \mathbb{R}$, then the spectrum below the energy $E_{1}^{D N}$ is empty. Our improvement is two-fold. Firstly, we generalize the first claim in the sense that we skip the condition on $k$. Secondly, we find a sufficient condition which guarantees the existence of spectrum below $E_{1}^{D N}$ even for some strips with $\alpha>0$. In addition to these substantial generalizations, we will derive the same result also for periodic waveguides. Let us summarize the spectral properties of $H^{D N}$ into the following theorem.

Theorem 4.4 (Dirichlet-Neumann case). Suppose $\langle\mathrm{H}\rangle$.

(i) If $k \not \equiv 0$, then any of the three conditions

(a) $k \in L^{1}(\mathbb{R})$ and $\alpha \equiv \int_{\mathbb{R}} k(s) d s \leq 0$

(b) $k$ is periodic

(c) $k_{-} \not \equiv 0$ and $d$ is small enough

is sufficient to guarantee that $\inf \sigma\left(H^{D N}\right)<E_{1}^{D N}$.

(ii) If $k_{-} \not \equiv 0$, then $\inf \sigma\left(H^{D N}\right) \geq E_{1}^{D N}$.

Consequently, if the strip is not straight but it is straight asymptotically, i.e. $\langle\mathrm{d}\rangle$, then any of the conditions (a) or (c) is sufficient to guarantee that $H^{D N}$ has at least one eigenvalue of finite multiplicity below its essential spectrum $\left[E_{1}^{D N}, \infty\right)$, i.e., $\sigma_{\mathrm{disc}}\left(H^{D N}\right) \neq \emptyset$. On the other hand, if the strip is asymptotically straight and $k_{-} \equiv 0$, then $\sigma\left(H^{D N}\right)=\sigma_{\mathrm{ess}}\left(H^{D N}\right)=\left[E_{1}^{D N}, \infty\right)$, i.e., $\sigma_{\mathrm{disc}}\left(H^{D N}\right)=\emptyset$.

Remark 4.1. The signs of $k(s)$ and the corresponding total bending angle $\alpha$ change after the change of arc-length parameter given by $s \mapsto-s$. It has to be stressed here that such a re-parameterization of the reference curve $\Gamma$ leads to another strip due to (3.1) and, consequently, there is no ambiguity in stating the spectral results on $H^{D N}$ in terms of the sign of $\alpha$ and $k$, see Figure 2 . 

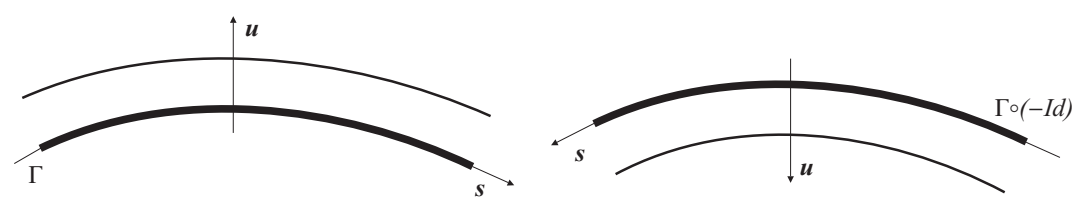

Figure 2. Inversion of orientation of the reference curve (given by the reparameterization $s \mapsto-s$ ). Thick lines denote the Dirichlet boundary condition, thin lines the Neumann one.

The sufficient conditions (a)-(c) of the first part of Theorem 4.4 are proved in Section 6.1. We refer to [17] for the original proof of the part (ii) (this proof is in fact very technical, based on a decomposition of $H^{D N}$ to the transverse basis (3.11) and the spectral analysis of an associated ordinary differential operator) and to [49] for a recent, simplified proof. A comparison of the condition (a) with the assumptions given in [17] is done in Remark 6.1.

Consider now a situation when the discrete spectrum of $H^{\iota}, \iota \in\{D, D N\}$, below the energy $E_{1}^{\iota}$ is not empty.

Although this paper in not intended to investigate the number of eigenvalues of $H^{\iota}$, let us point out the following remarkable property of $H^{D N}$ which we establish at the end of Section 6.1.

Proposition 4.1 (Number of bound states in the DN case). Suppose $\langle\mathrm{H}\rangle$ and $\langle\mathrm{d}\rangle$. If $k_{-} \not \equiv 0$ then

$$
\forall n \in \mathbb{N} \quad \exists d_{n}>0: \quad d<d_{n} \Longrightarrow N\left(H^{D N}\right) \geq n,
$$

where $N\left(H^{D N}\right)$ denotes the number of discrete eigenvalues of $H^{D N}$, counting multiplicity.

The number of bound states in thin strips is another property, which demonstrates a significant influence of the choice of boundary conditions on the spectrum. To see it, we recall that an upper SKN-type (cf $[73,54,67])$ bound on the number of bound states in thin Dirichlet strips was derived in [18, Sec. 2.3] and it showed that $N\left(H^{D}\right)$ is bounded from above by a finite constant which does not depend on the strip width $d$. On the other hand, Proposition 4.1 shows that $N\left(H^{D N}\right)$ can reach arbitrarily large value by shrinking the strip width to zero. 
The last objective of this paper is to estimate the distance between the bottom of the essential spectrum $E_{1}^{\iota}$ and the spectral threshold inf $\sigma\left(H^{\iota}\right)$ (which will represent the lowest eigenvalue since, for this problem, we restrict ourselves to the strips with curvature having compact support). We derive the following upper bounds (to the lowest eigenvalue), which are again qualitatively different for the Dirichlet and mixed Dirichlet-Neumann situations, respectively.

Theorem 4.5 (Estimates of the spectral threshold). Suppose $\langle\mathbf{H}\rangle$ and assume that $k$ has a compact support in an interval of width $2 s_{0}$.

(i) If $\alpha \leq 0$, then $\inf \sigma\left(H^{D N}\right) \leq E_{1}^{D N}-C^{D N}\left(s_{0}, d, \alpha\right)^{2} \alpha^{2}$, where

$$
C^{D N}\left(s_{0}, d, \alpha\right):=\sqrt{E_{1}^{D N}} \frac{\sqrt{3} / \pi}{1+\sqrt{1-\frac{3}{2} \frac{\alpha s_{0}}{d}+\frac{3}{4} \alpha^{2}\left(\frac{1}{2}+\frac{2}{\pi^{2}}\right)}} .
$$

(ii) $\inf \sigma\left(H^{D}\right) \leq E_{1}^{D}-C^{D}\left(s_{0}, d, \alpha\right)^{2} \alpha^{4}$, where

$$
C^{D}\left(s_{0}, d, \alpha\right):=\frac{2^{4}}{3^{3}} \frac{\sqrt{3} / \pi^{2}}{d\left(\frac{s_{0}}{d}-\frac{\alpha}{4}+\frac{2}{3 \pi}\right)} \frac{1}{1+\sqrt{1+\left(\frac{4 \alpha}{3 \pi}\right)^{2} \frac{4 s_{0}-\alpha d}{4 s_{0}-\alpha d+\frac{8 d}{3 \pi}}}} .
$$

These estimates are new in the theory of quantum waveguides and we derive them in Section 6. One can immediately see that for small total bending angles, the leading term in the estimate (i) is proportional to the second power of $\alpha$, while it is the fourth power of $\alpha$ in the estimate (ii). Another essential difference in our estimates appears in the limit case of thin strips. We discuss these interesting disparities in Remarks 6.3 and 6.4. We also compare there the result (ii) with the exact eigenvalue asymptotics obtained in [18] by perturbation methods applied to mildly curved or thin strips, respectively.

\section{$\S 5 . \quad$ Essential Spectrum}

This section is devoted to the proof of Theorem 4.1. It is achieved in two steps. Firstly, in Lemma 5.2, we employ a Neumann bracketing argument in order to show that the threshold of the essential spectrum does not descend below the energy $E_{1}^{\iota}$. Secondly, in Lemma 5.3 , we prove that all energies above $E_{1}^{\iota}$ belong to the spectrum by means of the following general characterization of essential spectrum which we have adopted from [15].

Lemma 5.1. $\quad$ Let $H$ be a non-negative self-adjoint operator in a complex Hilbert space $\mathcal{H}$ and $Q$ be the associated quadratic form. Then $\eta \in \sigma_{\mathrm{ess}}(H)$ if 
and only if

$$
\exists\left\{\psi_{n}\right\}_{n=1}^{\infty} \subset \operatorname{Dom} Q: \begin{cases}\text { (i) } & \forall n \in \mathbb{N} \backslash\{0\}:\left\|\psi_{n}\right\|=1, \\ \text { (ii) } & \psi_{n} \underset{n \rightarrow \infty}{\longrightarrow} 0 \text { in } \mathcal{H}, \\ \text { (iii) } & (H-\eta) \psi_{n} \underset{n \rightarrow \infty}{\longrightarrow} 0 \quad \text { in }(\operatorname{Dom} Q)^{*} .\end{cases}
$$

Here $(\operatorname{Dom} Q)^{*}$ denotes the dual of the space $\operatorname{Dom} Q$. We note that $H+1$ : $\operatorname{Dom} Q \rightarrow(\operatorname{Dom} Q)^{*}$ is an isomorphism and

$$
\|\psi\|_{-1}:=\|\psi\|_{(\operatorname{Dom} Q)^{*}}=\sup _{\phi \in \operatorname{Dom} Q \backslash\{0\}} \frac{|(\phi, \psi)|}{\|\phi\|_{1}}
$$

with

$$
\|\phi\|_{1}:=\sqrt{Q[\phi]+\|\phi\|^{2}} .
$$

Lemma 5.1 is proved in a quite similar fashion as the Weyl criterion, [75, Thm. 7.24]. The advantage of the present characterization is that it requires to find a sequence from the form domain of $H$ only, and not from Dom $H$ as it is required by the Weyl criterion. Moreover, in order to check the limit from (iii), it is still sufficient to consider the operator $H$ in the form sense, i.e. we will not need to assume that $\left(G_{i j}\right)$ is differentiable in our case.

We start by an estimate on the threshold of the essential spectrum.

Lemma 5.2. If $\langle\mathrm{d}\rangle$ holds true, then $\inf \sigma_{\mathrm{ess}}\left(H^{\iota}\right) \geq E_{1}^{\iota}$.

Proof. Since the curvature vanishes at infinity, for any fixed $\epsilon>0$, there exists $s_{\epsilon}$ such that

$$
\forall(s, u) \in \Omega_{\mathrm{ext}}:(1-d \epsilon) \leq 1-u k(s) \leq(1+d \epsilon),
$$

where $\Omega_{\text {ext }}:=\Omega_{0} \backslash \bar{\Omega}_{\text {int }}$ with $\Omega_{\text {int }}:=\left(-s_{\epsilon}, s_{\epsilon}\right) \times I$. Denote by $H_{N}^{\iota}$ the operator $H^{\iota}$ with a supplementary Neumann boundary condition imposed on the two segments $\left\{ \pm s_{\epsilon}\right\} \times I$, that is, the operator associated with the form $Q_{N}^{\iota}:=Q_{N}^{\iota, \text { int }} \oplus Q_{N}^{\iota, \text { ext }}$, where

$$
Q_{N}^{\iota, \omega}[\psi]:=\left(\psi_{, i}, G^{i j} \psi_{, j}\right)_{L^{2}\left(\Omega_{\omega}, d \Omega\right)},
$$

$\operatorname{Dom} Q_{N}^{D, \omega}:=\left\{\psi \in W^{1,2}\left(\Omega_{\omega}, d \Omega\right) \mid \psi(s, 0)=\psi(s, d)=0\right.$ for a.e. $\left.s \in \mathbb{R} \cap \bar{\Omega}_{\omega}\right\}$,

$\operatorname{Dom} Q_{N}^{N, \omega}:=W^{1,2}\left(\Omega_{\omega}, d \Omega\right)$,

$\operatorname{Dom} Q_{N}^{D N, \omega}:=\left\{\psi \in W^{1,2}\left(\Omega_{\omega}, d \Omega\right) \mid \psi(s, 0)=0\right.$ for a.e. $\left.s \in \mathbb{R} \cap \bar{\Omega}_{\omega}\right\}$, 
for $\omega \in\{$ int, ext $\}$. Since $H^{\iota} \geq H_{N}^{\iota}$ and the spectrum of the operator associated with $Q_{N}^{\iota, \text { int }}$ is purely discrete, of [13, Chap. 7], the minimax principle gives the estimate

$$
\inf \sigma_{\text {ess }}\left(H^{\iota}\right) \geq \inf \sigma_{\text {ess }}\left(H_{N}^{\iota, \text { ext }}\right) \geq \inf \sigma\left(H_{N}^{\iota, \text { ext }}\right),
$$

where $H_{N}^{\iota, \text { ext }}$ denotes the operator associated with $Q_{N}^{\iota, \text { ext }}$. Neglecting the nonnegative "longitudinal" part of the Laplacian in (3.8) (i.e. the term where one sums over $i=j=1$ ) and using the estimates (5.2), we arrive easily at the following lower bound

$$
H_{N}^{\iota, \text { ext }} \geq \frac{1-d \epsilon}{1+d \epsilon} E_{1}^{\iota} \quad \text { in } \quad L^{2}\left(\Omega_{\mathrm{ext}}, d \Omega\right)
$$

which holds in the form sense (see also proof of Theorem 4.1 in [20]). The claim then follows by the fact that $\epsilon$ can be chosen arbitrarily small.

Remark 5.1 (Neumann case). Since $E_{1}^{N}=0$ and $H^{N}$ is a non-negative operator, the statement of Lemma 5.2 holds trivially true for the Neumann boundary conditions, i.e., $\iota=N$, even without the assumption $\langle\mathrm{d}\rangle$.

Example 5.1 (Periodic waveguides). The periodic strip (i.e. assumption $\langle d\rangle$ is not obeyed) is the simplest example for which

$$
\inf \sigma_{\text {ess }}\left(H^{\iota}\right)<E_{1}^{\iota}, \quad \iota \in\{D, D N\} .
$$

Let $k \not \equiv 0$ be a periodic function of a period $L>0$, i.e., $\forall s \in \mathbb{R}: k(s+L)=k(s)$, and such that the hypothesis $\langle\mathrm{H}\rangle$ holds true for some $d>0$. Then the operator $H^{\iota}$ is invariant with respect to the transformation $s \mapsto s+j L$ for every $j \in \mathbb{Z}$, which implies that there is no discrete eigenvalue in its spectrum, i.e. $\sigma\left(H^{\iota}\right)=$ $\sigma_{\text {ess }}\left(H^{\iota}\right)$. However, Theorems 4.3 and 4.4 state that $\inf \sigma\left(H^{\iota}\right)<E_{1}^{\iota}$.

According to a common belief, second order elliptic differential operators with sufficiently regular periodic coefficients should not have degenerate bands in their spectra, or, in other words, their spectra should be purely absolutely continuous (see [74] and references therein). An elegant rigorous proof of this fact for Dirichlet and Neumann periodic waveguides was given by E. Shargorodsky and A. Sobolev in [74] (cf also [4] for thin Dirichlet tubes).

The precedent Lemma 5.2 together with the following one establish Theorem 4.1.

Lemma 5.3. If $\langle\mathrm{d}\rangle$ holds true, then $\sigma_{\mathrm{ess}}\left(H^{\iota}\right) \supseteq\left[E_{1}^{\iota}, \infty\right)$. 
Proof. Let $n \in \mathbb{N} \backslash\{0\}$. We shall construct a sequence $\left\{\psi_{n}^{\iota}\right\}$ satisfying (i)-(iii) of Lemma 5.1 with $\eta^{\iota}:=\lambda^{2}+E_{1}^{\iota}$ for all $\lambda \in \mathbb{R}$. We start with the following family of functions

$$
\hat{\psi}_{n}^{\iota}(s, u):=\varphi_{n}(s) \chi_{1}^{\iota}(u) e^{i \lambda s}
$$

where $\chi_{1}^{\iota}$ is the lowest transverse-mode function (3.11) if $\iota \in\{D, D N\}$, or (3.12) if $\iota=N$, respectively, and $\varphi_{n}(s):=\varphi\left(n^{-1} s-n\right)$ with $\varphi$ being a non-zero $C^{\infty}$ smooth function with a compact support in $(-1,1)$. Note that $\operatorname{supp} \varphi_{n} \subset$ $\left(n^{2}-n, n^{2}+n\right)$ and, consequently, the sequence $\left\{\varphi_{n}\right\}$ is "localized at $+\infty$ " for large $n$. It is clear that $\hat{\psi}_{n}^{\iota}$ belongs to the form domain of $H^{\iota}$. Since it is not normalized in $\mathcal{H}$, we introduce $\psi_{n}^{\iota}:=\hat{\psi}_{n}^{\iota} /\left\|\hat{\psi}_{n}^{\iota}\right\|$. Hereafter we shall use the equivalence of the norms $\|\cdot\|$ and $\|\cdot\|_{L^{2}\left(\Omega_{0}\right)}$, which follows by (3.3). In particular, one has

$$
C_{-}\left\|\varphi_{n}\right\|_{L^{2}(\mathbb{R})}^{2} \leq\left\|\hat{\psi}_{n}^{\iota}\right\|^{2} \leq C_{+}\left\|\varphi_{n}\right\|_{L^{2}(\mathbb{R})}^{2}
$$

due to the normalization of $\chi_{1}^{\iota}$.

The point (ii) of Lemma 5.1 requires that $\left(\phi, \psi_{n}^{\iota}\right) \rightarrow 0$ as $n \rightarrow \infty$ for all $\phi \in \mathcal{H}$. Since $\left\{\psi_{n}^{\iota}\right\}$ is bounded in $\mathcal{H}$, it is enough to show the limit for all $\phi \in C_{0}^{\infty}\left(\Omega_{0}\right)$, a dense subset of $\mathcal{H}$. However, the latter follows at once because $\phi$ and $\psi_{n}^{\iota}$ will have disjoint supports for $n$ large enough.

Hence, it remains to check that $\left\|\left(H^{\iota}-\eta^{\iota}\right) \psi_{n}\right\|_{-1} \rightarrow 0$ as $n \rightarrow \infty$. Employing the diagonal form (3.2) of the metric tensor, we can split the Hamiltonian (3.8) into a sum of two parts, $H^{\iota}=H_{1}^{\iota}+H_{2}^{\iota}$, where $H_{i}^{\iota}, i \in\{1,2\}$, corresponds to the term with $G^{i i}$ in (3.8). This decomposition leads to the trivial bound

$$
\left\|\left(H^{\iota}-\eta^{\iota}\right) \psi_{n}^{\iota}\right\|_{-1} \leq\left\|\left(H_{1}^{\iota}-\lambda^{2}\right) \psi_{n}^{\iota}\right\|_{-1}+\left\|\left(H_{2}^{\iota}-E_{1}^{\iota}\right) \psi_{n}^{\iota}\right\|_{-1} .
$$

We will show that the norms at the r.h.s. of this inequality tends to zero as $n \rightarrow \infty$ separately. Denote $\|f\|_{\infty, n}:=\sup \left\{|f(s, u)| \mid(s, u) \in \operatorname{supp} \varphi_{n} \times I\right\}$.

An explicit calculation using (3.3) and the fact that $\chi_{1}^{\iota}$ is an eigenfunction of $-\Delta_{\iota}^{I}$ corresponding to the energy $E_{1}^{\iota}$ yields

$$
\left|\left(\phi,\left(H_{2}^{\iota}-E_{1}^{\iota}\right) \hat{\psi}_{n}^{\iota}\right)\right|=\left|\left(\phi, k \hat{\psi}_{n, 2}^{\iota}\right)_{L^{2}\left(\Omega_{0}\right)}\right| \leq C_{-}^{-1} \sqrt{E_{1}^{\iota}}\|k\|_{\infty, n}\|\phi\|\left\|\hat{\psi}_{n}^{\iota}\right\|
$$

for all $\phi \in \operatorname{Dom} Q^{\iota}$. Consequently, the second term at the r.h.s. of (5.4) goes to zero as $n \rightarrow \infty$ by the assumption $\langle\mathrm{d}\rangle$. 
A little more toilsome but still direct calculation yields

$$
\begin{aligned}
\left(\phi,\left(H_{1}^{\iota}-\lambda^{2}\right) \hat{\psi}_{n}^{\iota}\right)= & \lambda^{2}\left(\phi,\left(1-G^{\frac{1}{2}}\right) \hat{\psi}_{n}^{\iota}\right)_{L^{2}\left(\Omega_{0}\right)} \\
& +\left(\phi, 1,\left(G^{\frac{1}{2}} G^{11}-1\right)\left(\dot{\varphi}_{n}+i \lambda \varphi_{n}\right) \chi_{1}^{\iota} e^{i \lambda s}\right)_{L^{2}\left(\Omega_{0}\right)} \\
& -\left(\phi,\left(\ddot{\varphi}_{n}+2 i \lambda \dot{\varphi}_{n}\right) \chi_{1}^{\iota} e^{i \lambda s}\right)_{L^{2}\left(\Omega_{0}\right)}
\end{aligned}
$$

for all $\phi \in \operatorname{Dom} Q^{\iota}$. Estimating all the terms at the r.h.s. of this equality in the same way as in (5.5), it is enough to show that the following sequences

$$
\left\|1-G^{\frac{1}{2}}\right\|_{\infty, n}, \quad\left\|G^{\frac{1}{2}} G^{11}-1\right\|_{\infty, n}, \quad \frac{\left\|\dot{\varphi}_{n}\right\|_{L^{2}(\mathbb{R})}}{\left\|\varphi_{n}\right\|_{L^{2}(\mathbb{R})}}, \quad \frac{\left\|\ddot{\varphi}_{n}\right\|_{L^{2}(\mathbb{R})}}{\left\|\varphi_{n}\right\|_{L^{2}(\mathbb{R})}},
$$

has the zero limit as $n \rightarrow \infty$. However, this is evident for the first and second ones by virtue of (3.2) and $\langle d\rangle$, while for the rest it follows by the definition of the sequence $\left\{\varphi_{n}\right\}$.

If the strip is asymptotically straight, i.e. $\langle\mathrm{d}\rangle$, then $\sigma\left(H^{N}\right)=[0, \infty)$ by Theorem 4.1, (3.10) and non-negativity of $H^{N}$; see also Theorem 4.2. We conclude this section by proving the following result about the spectral threshold of the operators $H^{D}$ and $H^{D N}$.

Proposition 5.1. $\quad$ Suppose $\langle\mathrm{H}\rangle$. If the strip obeys $\langle\mathrm{d}\rangle$, then

$$
\inf \sigma\left(H^{\iota}\right)>0 \quad \text { for } \quad \iota \in\{D, D N\} \text {. }
$$

Proof. We have $H^{\iota} \geq 0$ and $E_{1}^{\iota}>0$. By virtue of Theorem 4.1, it is enough to prove that $0 \notin \sigma_{\mathrm{p}}\left(H^{\iota}\right)$. Assume that there exists $\psi \in \operatorname{Dom} H^{\iota}$ such that $H^{\iota} \psi=0$. Then $\psi \in \operatorname{Dom} Q^{\iota}$ and $0=\left(\psi, H^{\iota} \psi\right)=Q^{\iota}[\psi] \equiv$ $\int_{\Omega_{0}} \bar{\psi}_{, i} G^{i j} \psi_{, j} d \Omega$ with $\left(G_{i j}\right)$ being a strictly positive definite matrix, hence $\psi=0$ a.e.

Actually, stronger lower bounds to inf $\sigma\left(H^{D}\right)$ were derived in $[1,25]$.

\section{§6. Curvature-induced Spectrum}

Now we will be interested in the proofs concerning the existence and properties of the spectrum of $H^{\iota}$ below the energy $E_{1}^{\iota}$. Since $H^{N}$ is a non-negative operator and $E_{1}^{N}=0$, only the situations $\iota \in\{D, D N\}$ are relevant here, however, we do not exclude the Neumann case from the preliminary considerations here in order to establish a minor result contained in Proposition 6.1 below. 
All the proofs of the following subsections are based on the variational strategy of finding a trial function $\psi^{\iota}$ from the form domain of $H^{\iota}$ such that

$$
Q_{1}^{\iota}\left[\psi^{\iota}\right]:=Q^{\iota}\left[\psi^{\iota}\right]-E_{1}^{\iota}\left\|\psi^{\iota}\right\|^{2}<0 .
$$

We construct such a trial function by modifying the generalized eigenfunction (3.11) of energy $E_{1}^{\iota}$ for the straight strip. This idea goes back to J. Goldstone and R. L. Jaffe, [52]; see also [18, 20, 17, 58].

As a preliminary, let us express the form (6.1) in the situation where the variables are separated in the following way:

$$
\psi^{\iota}(s, u):=\varphi(s) \chi_{1}^{\iota}(u)
$$

where $\chi_{1}^{\iota}$ is the first transverse mode (3.11) or (3.12) and $\varphi$ is a suitable function from $W^{1,2}(\mathbb{R})$. In view of $(3.3)$, it is clear that $\psi^{\iota}$ belongs to $\operatorname{Dom} Q^{\iota}$, given by (3.5), (3.6) or (3.7), respectively. An explicit calculation yields

$$
Q_{1}^{\iota}\left[\psi^{\iota}\right]=\left(\dot{\varphi},\left\langle G^{-\frac{1}{2}}\right\rangle_{\iota} \dot{\varphi}\right)_{L^{2}(\mathbb{R})}+\frac{1}{2}\left[\chi_{1}^{\iota}(d)^{2}-\chi_{1}^{\iota}(0)^{2}\right](\varphi, k \varphi)_{L^{2}(\mathbb{R})},
$$

where $\langle\cdot\rangle_{\iota}$ denotes the expectation with respect to $\chi_{1}^{\iota}$, i.e.

$$
\langle f\rangle_{\iota}:=\int_{I} f(\cdot, u) \chi_{1}^{\iota}(u)^{2} d u
$$

with $f \in L^{\infty}\left(\Omega_{0}\right)$. It is clear from (3.11) and (3.12) that the second term at the r.h.s. of (6.3) is absent for $\iota \in\{D, N\}$, while $\chi_{1}^{D N}(d)=\sqrt{2 / d}$ and $\chi_{1}^{D N}(0)=0$.

\section{§6.1. The existence}

Proof of Theorem 4.3 and Theorem 4.4, condition (a). We set

$$
\psi_{n}^{\iota}(s, u):=\varphi(s ; n) \chi_{1}^{\iota}(u),
$$

where $\varphi: \mathbb{R} \times(0, \infty) \rightarrow[0,1]$ is supposed to satisfy:

(i) $\forall n \in(0, \infty): \varphi(\cdot ; n) \in W^{1,2}(\mathbb{R})$,

(ii) $\varphi(s ; n) \underset{n \rightarrow \infty}{\longrightarrow} 1$ for a.e. $s \in \mathbb{R}$,

(iii) $\|\varphi, 1(\cdot ; n)\|_{L^{2}(\mathbb{R})} \underset{n \rightarrow \infty}{\longrightarrow} 0$,

that is, $\varphi$ is a suitable mollifier of 1 (for an example of such a function, see (6.12) below). Substituting this trial function to (6.3), we get

$$
Q_{1}^{D}\left[\psi_{n}\right] \underset{n \rightarrow \infty}{\longrightarrow} 0, \quad Q_{1}^{N}\left[\psi_{n}\right] \underset{n \rightarrow \infty}{\longrightarrow} 0, \quad Q_{1}^{D N}\left[\psi_{n}\right] \underset{n \rightarrow \infty}{\longrightarrow} \frac{\alpha}{d}
$$


where $\alpha$ is the total bending angle (4.1). The limits hold true by virtue of the required properties of $\varphi$, the fact that $\left\langle G^{-\frac{1}{2}}\right\rangle_{\iota}$ are bounded functions and, in the case $\iota=D N$, also by the dominated convergence theorem. That is why we need to assume in addition that $k$ is integrable for $\iota=D N$. Consequently, if $\alpha$ is strictly negative, then there exists a finite $n_{0}>0$ such that $Q_{1}^{D N}\left[\psi_{n_{0}}\right]<0$ and the proof for $\iota=D N$ is finished in this case.

To obtain the result for $\iota=D N$ in the limit case $\alpha=0$, and for any Dirichlet strip, we modify the function $\psi_{n}^{\iota}$, in a curved part of the waveguide. We define

$$
\psi_{n, \varepsilon}^{\iota}(s, u):=\psi_{n}^{\iota}(s, u)+\varepsilon \phi(s) v^{\iota}(u) \chi_{1}^{\iota}(u), \quad \iota \in\{D, D N\}
$$

where $\varepsilon \in \mathbb{R}, \phi \in W^{1,2}(\mathbb{R})$ is a real, non-negative, non-zero function with compact support contained in a bounded interval in $\mathbb{R}$ where $k$ is not zero and does not change sign (such an interval surely exists because $k \neq 0$ and is continuous), and $v^{D}(u):=-2 u / d$ and $v^{D N}(u):=1$. The family $\left\{\psi_{n, \varepsilon}^{\iota}\right\}$ is a subset of $\operatorname{Dom} Q^{\iota}$ and we can write

$$
Q_{1}^{\iota}\left[\psi_{n, \varepsilon}^{\iota}\right]=Q_{1}^{\iota}\left[\psi_{n}^{\iota}\right]+2 \varepsilon Q_{1}^{\iota}\left(\phi v^{\iota} \chi_{1}^{\iota}, \psi_{n}^{\iota}\right)+\varepsilon^{2} Q_{1}^{\iota}\left[\phi v^{\iota} \chi_{1}^{\iota}\right] .
$$

The last term at the r.h.s. of (6.7) does not depend on $n$, while the first one tends to zero as $n \rightarrow \infty$ by (6.5). An explicit calculation of the central term gives $(c f(6.3)$ for $\iota=D N)$

$$
Q_{1}^{\iota}\left(\phi v^{\iota} \chi_{1}^{\iota}, \psi_{n}\right)=\left(\dot{\phi},\left\langle v^{\iota} G^{-\frac{1}{2}}\right\rangle_{\iota}, \dot{\varphi}_{n}\right)_{L^{2}(\mathbb{R})}+\frac{1}{d}\left(\phi, k \varphi_{n}\right)_{L^{2}(\mathbb{R})},
$$

where we have denoted $\varphi_{n}:=\varphi(\cdot ; n)$ and $\dot{\varphi}_{n}:=\varphi_{, 1}(\cdot ; n)$. Using then the properties of the function $\varphi$ together with the dominated convergence theorem (notice that $\phi k \in L^{1}(\mathbb{R})$ ), we have

$$
Q_{1}^{\iota}\left[\psi_{n, \varepsilon}^{\iota}\right] \underset{n \rightarrow \infty}{\longrightarrow} \frac{2}{d} \varepsilon(\phi, k)_{L^{2}(\mathbb{R})}+\varepsilon^{2} Q_{1}^{\iota}\left[\phi v^{\iota} \chi_{1}^{\iota}\right] .
$$

Since the integral $(\phi, k)_{L^{2}(\mathbb{R})}$ is non-zero by the construction of $\phi$, we can take $\varepsilon$ sufficiently small and of an appropriate sign so that the sum of the last two terms at the r.h.s. of (6.8) is negative, and then choose $n$ sufficiently large so that $Q_{1}^{\iota}\left[\psi_{n, \varepsilon}^{\iota}\right]<0$.

The intermediate results (6.5) of the precedent proof give the following upper bounds to the spectral threshold of $H^{\iota}$ :

Proposition 6.1. Suppose $\langle\mathrm{H}\rangle$. One has

(i) $\inf \sigma\left(H^{\iota}\right) \leq E_{1}^{\iota} \quad$ for $\iota \in\{D, N\}$; 
(ii) $\inf \sigma\left(H^{D N}-\frac{k(s)}{d(1-u k(s))}\right) \leq E_{1}^{D N} \quad$ provided $k \in L^{1}(\mathbb{R})$.

Actually, in view of Theorem 4.3, a stronger result than (i) holds for any Dirichlet strip. The assertion (i) for the Neumann case, together with the fact that $H^{N}$ is non-negative, establishes the first claim of Theorem 4.2.

Remark 6.1 (Condition (a) of Theorem 4.4 vs the assumptions in [17]). The non-positivity of the total bending angle, i.e. $\alpha \leq 0$, is a nice sufficient condition which guarantees the existence of geometrically induced spectrum for $H^{D N}$. This was established already in [17] under the additional hypothesis that " $k$ is non-positive everywhere outside of some bounded interval". Since the latter is not assumed in this paper, we extend significantly the class of admissible geometries. Nevertheless, in order to justify the use of the dominated convergence theorem, we need to assume that " $k$ is integrable" instead; $c f$ the condition (a) of Theorem 4.4. Hence, a natural question is to ask whether the assumptions in [17] may after all present an alternative criterion which is not contained in our condition (a). The answer is negative due to the following (purely geometrical) result, which can be easily shown using the so-called "Umlaufsatz", [56, Thm. 2.2.1]:

Lemma 6.1. Let $\Gamma$ be an infinite plane $C^{2}$-smooth curve of bounded curvature $k$. If there exists a compact $\Gamma_{c} \subset \Gamma$ such that $\left|\int_{\Gamma_{a}} k\right|>2 \pi$ for any compact $\Gamma_{a}$ obeying $\Gamma_{c} \subseteq \Gamma_{a} \subset \Gamma$, then any tubular neighbourhood of $\Gamma$ overlaps.

That is, any reference curve satisfying the assumptions of [17] but having a non-integrable curvature leads to a violation of the basic hypothesis $\langle\mathrm{H}\rangle$ (which is assumed in [17] as well).

Proof of Theorem 4.4, condition (b). Let $L>0$ be the period of $k$, i.e., $\forall s \in \mathbb{R}: k(s+L)=k(s)$. We take the trial function of the form

$$
\psi_{n, \varepsilon}^{D N}(s, u):=\varphi_{n}(s)(1+\varepsilon \phi(s)) \chi_{1}^{D N}(u),
$$

cf (6.6), where the functions $\varphi_{n}$ and $\phi$ are defined as follows. Let $\varphi_{1} \in C_{0}^{\infty}(\mathbb{R})$ be a real function with the support inside the interval $(-L, 2 L)$ which is equal to 1 on the period cell $(0, L)$. We set, for any $n \in \mathbb{N} \backslash\{0\}$,

$$
\varphi_{n}(s):= \begin{cases}\varphi_{1}(s) & \text { if } \quad s \in(-\infty, L), \\ 1 & \text { if } s \in[L, n L], \\ \varphi_{1}(s-(n-1) L) & \text { if } \quad s \in(n L,+\infty) .\end{cases}
$$


Let $\phi \in C^{\infty}(\mathbb{R})$ be non-negative, $L$-periodic, and such that $\operatorname{supp} \phi \uparrow(0, L)$ is contained in an interval where $k$ is not zero and does not change sign. Then $(\phi, k)_{L^{2}((0, L))} \neq 0$. Finally, let $\varepsilon \in \mathbb{R}$ be chosen in such a way that $(c f(6.8))$

$$
\begin{aligned}
A & :=\left(\psi_{1, \varepsilon}^{D N},\left(H^{D N}-E_{1}^{D N}\right) \psi_{1, \varepsilon}^{D N}\right)_{L^{2}((0, L) \times I, d \Omega)} \\
& =\frac{2}{d} \varepsilon(\phi, k)_{L^{2}((0, L))}+\varepsilon^{2}\left(\phi \chi_{1}^{D N},\left(H^{D N}-E_{1}^{D N}\right) \phi \chi_{1}^{D N}\right)_{L^{2}((0, L) \times I, d \Omega)}
\end{aligned}
$$

is negative. By virtue of the definition of $\varphi_{1}$ and the fact that $\int_{0}^{L} k(s) d s=0$ (cf Lemma 6.1), it is clear that

$$
Q_{1}^{D N}\left[\psi_{1, \varepsilon}^{D N}\right]=A+B
$$

where $B$ is defined as the integral at the first line of (6.9), however, with the range of integration being the set $((-L, 0) \cup(L, 2 L)) \times I$. Using the periodicity of the coefficients of $H^{D N}$ together with the definition of $\varphi_{n}$, we continue by induction and arrive at the identity

$$
\forall n \in \mathbb{N} \backslash\{0\}: \quad Q_{1}^{D N}\left[\psi_{n, \varepsilon}^{D N}\right]=n A+B,
$$

which becomes negative for $n$ sufficiently large.

Remark 6.2 (Integrability of $k$ ). If $k \neq 0$ is periodic, then the curvature is not integrable. However, one has for every $n \in \mathbb{N}, \int_{-n L}^{n L} k(s) d s=0$ due to the periodicity ( $c f$ Lemma 6.1). This indicates that the requirement $k \in L^{1}(\mathbb{R})$ in the condition (a) of Theorem 4.4 may be rather a technical hypothesis.

Proof of Theorem 4.4, condition (c). We take the trial function $\psi^{D N}$ of the form (6.2). Since $k$ is continuous and $k_{-} \not \equiv 0$, there exists an interval $J \subset \mathbb{R}$, such that $k(s)<0$ for all $s \in J$. Choosing $\varphi \in W^{1,2}(\mathbb{R})$ such that $\operatorname{supp} \varphi \subseteq \bar{J}$ and substituting it to (6.3), obvious estimates yield

$$
Q_{1}^{D N}\left[\psi^{D N}\right] \leq\|\dot{\varphi}\|_{L^{2}(J)}^{2}+\frac{1}{d} \int_{J}|\varphi(s)|^{2} k(s) d s .
$$

The second term at the r.h.s. of the last inequality is obviously negative, while the first one does not depend on $d$. Hence for all $d$ sufficiently small their sum is negative.

Proof of Proposition 4.1. The claim is trivial for $n=0$. Let us fix an integer $n \in \mathbb{N} \backslash\{0\}$. We shall find a critical width $d_{n}$ such that for 
all $d<d_{n}$, there are at least $n$ discrete eigenvalues in the spectrum of $H^{D N}$, counting multiplicity. As in the proof of Theorem 4.4, condition (c), let $J \subset \mathbb{R}$ be a bounded interval such that $k(s)<0$ for all $s \in J$. We set $s_{0}:=\inf J$ and $s_{j}:=s_{0}+j|J| / n$ for every $j \in\{1, \ldots, n\}$. Let $\varphi_{0}$ be a non-zero function from $W^{1,2}(\mathbb{R})$ such that $\operatorname{supp} \varphi_{0} \subset\left(s_{0}, s_{1}\right)$. We define for every $j \in\{1, \ldots, n\}$ and $s \in \mathbb{R}$,

$$
\begin{aligned}
N_{j}^{-2} & :=\int_{s_{j-1}}^{s_{j}}\left|\varphi_{0}\left(s_{0}+s-s_{j-1}\right)\right|^{2}\left\langle G^{\frac{1}{2}}\right\rangle_{D N}(s) d s, \\
\varphi_{j}(s) & :=N_{j} \varphi_{0}\left(s_{0}+s-s_{j-1}\right) .
\end{aligned}
$$

Putting $\psi_{j}^{D N}(s, u):=\varphi_{j}(s) \chi_{1}^{D N}(u)$ for every $j \in\{1, \ldots, n\}, c f(6.2)$, we get an orthonormal basis of a subspace of Dom $Q^{D N}$. Moreover, $Q^{D N}\left(\psi_{j}^{D N}, \psi_{\ell}^{D N}\right)=0$ whenever $j \neq \ell$ because $\varphi_{j}$ and $\varphi_{\ell}$ have disjoint supports. Therefore, it follows by [13, Lemma 4.5.4] and Theorem 4.1 that a sufficient condition for $H^{D N}$ to have at least $n$ discrete eigenvalue is $Q^{D N}\left[\psi_{j}^{D N}\right]<E_{1}^{D N}$, i.e. $Q_{1}^{D N}\left[\psi_{j}^{D N}\right]<0$, for every $j \in\{1, \ldots, n\}$. However, according to (6.10),

$$
Q_{1}^{D N}\left[\psi_{j}^{D N}\right] \leq N_{j}^{2}\left\|\dot{\varphi}_{0}\right\|_{L^{2}(\mathbb{R})}^{2}+\frac{N_{j}^{2}}{d} \int_{s_{j-1}}^{s_{j}}\left|\varphi_{0}\left(s_{0}+s-s_{j-1}\right)\right|^{2} k(s) d s .
$$

The r.h.s. of the last inequality is obviously negative for all $j \in\{1, \ldots, n\}$ provided that $d<d_{n}$ with

$$
d_{n}:=\min _{j \in\{1, \ldots, n\}} \frac{1}{\left\|\dot{\varphi}_{0}\right\|_{L^{2}(\mathbb{R})}^{2}} \int_{s_{0}}^{s_{1}}\left|\varphi_{0}(s)\right|^{2}\left|k\left(s-s_{0}+s_{j-1}\right)\right| d s .
$$

\section{§6.2. The estimates on the spectral threshold}

Throughout this subsection, we consider only $\iota \in\{D, D N\}$. Obviously,

$$
\inf \sigma\left(H^{\iota}\right)-E_{1}^{\iota}=\inf _{\psi \in \operatorname{Dom} Q^{\iota}} \frac{Q_{1}^{\iota}[\psi]}{\|\psi\|^{2}} \leq \inf _{\psi \in T^{\iota}} \frac{Q_{1}^{\iota}[\psi]}{\|\psi\|^{2}},
$$

where $T^{\iota}$ is an arbitrary subset of Dom $Q^{\iota}$. Our strategy will be to choose a suitable $T^{\iota}$ and then explicitly find the infimum of the quotient at the r.h.s. of $(6.11)$.

In Theorem 4.5, the curvature is supposed to have a compact support contained in an interval of width $2 s_{0}$; without loss of generality we may assume that the reference curve is parameterized in such a way that $\operatorname{supp} k \subseteq\left[-s_{0}, s_{0}\right]$. 
Proof of Theorem 4.5, part (i). Let $\psi_{n, c}(s, u):=\varphi_{c}(s ; n) \chi_{1}^{D N}(u)$ be the trial function from the beginning of the proof of the condition (a) of Theorem 4.4 in Section 6.1 with the mollifier $\varphi_{c}(\cdot ; n)$ given explicitly by

$$
\varphi_{c}(s ; n):=\left\{\begin{array}{lll}
1 & \text { if } \quad|s| \in[0, n), \\
(c n-|s|) /((c-1) n) & \text { if } \quad|s| \in[n, c n), \\
0 & \text { if } \quad|s| \in[c n, \infty), &
\end{array}\right.
$$

We set $T^{D N}:=\left\{\psi_{n, c} \mid n \geq s_{0}, c>1\right\}$. An easy calculation yields

$$
Q_{1}^{D N}\left[\psi_{n, c}\right]=\frac{2}{(c-1) n}+\frac{\alpha}{d}, \quad\left\|\psi_{n, c}\right\|^{2}=\frac{2}{3}(c+2) n-\alpha\langle u\rangle,
$$

where

$$
\langle u\rangle:=\int_{I} u \chi_{1}^{D N}(u)^{2} d u=d\left(\frac{1}{2}+\frac{2}{\pi^{2}}\right) .
$$

Hence, denoting by $f(n, c)$ the quotient at the r.h.s. of $(6.11)$, we have

$$
f(n, c)=\frac{\frac{2}{c-1}+\frac{\alpha}{d} n}{\frac{2}{3}(c+2) n^{2}-\alpha\langle u\rangle n} .
$$

Now we shall seek the infimum of the continuous function $f$ in the region $\left[s_{0}, \infty\right) \times(1, \infty)$; the result establishes the bound from Theorem 4.5.

One can directly check that there is no local minimum of the function $f$ in the interior of its domain, i.e. for every point $(n, c) \in\left(s_{0}, \infty\right) \times(1, \infty)$, $f_{, 1}(n, c) \neq 0$ or $f_{, 2}(n, c) \neq 0$. Thus the problem reduces to the study of the behaviour of $f$ on the boundary set $\left\{s_{0}\right\} \times(1, \infty)$ and its limits as $n \rightarrow \infty$, $c \rightarrow 1$ and $c \rightarrow \infty$, respectively. The function $f\left(s_{0}, \cdot\right)$ reaches its (negative) local minimum

$$
f\left(s_{0}, c_{+}\right)=\frac{-3 \alpha^{2} / d^{2}}{4\left(1+\sqrt{1-\frac{3}{2} \alpha s_{0} / d+\frac{3}{4} \alpha^{2}\langle u\rangle / d}\right)^{2}}
$$

for

$$
c_{+}:=-\frac{2 d}{\alpha s_{0}}+1-\frac{d}{\alpha s_{0}} \sqrt{-6 \frac{\alpha s_{0}}{d}+4+3 \frac{\alpha^{2}\langle u\rangle}{d}} .
$$

Using the estimate $f(n, c) \geq \alpha /(2 d n)$, we obtain

$$
\liminf _{n \rightarrow \infty} f(n, c) \geq 0
$$

uniformly in $c$. Hence, there exists a (finite) $n_{0}>s_{0}$ such that for every $n>n_{0}$ holds true $f(n, c) \geq f\left(s_{0}, c_{+}\right)$(recall that $f\left(s_{0}, c_{+}\right)<0, c f(6.14)$ ) uniformly 
in $c$. Therefore since we seek the infimum of $f$ we can consider only $n \in\left[s_{0}, n_{0}\right]$ in the rest of the proof. However, for those values of $n$ we have

$$
f(n, c) \geq \frac{6}{(c-1)\left(2(c+2) n_{0}^{2}-3 \alpha\langle u\rangle n_{0}\right)}+\frac{3 \alpha / d}{2(c+2) n-3 \alpha\langle u\rangle}
$$

and since

$$
\lim _{c \rightarrow 1} \frac{6}{(c-1)\left(2(c+2) n_{0}^{2}-3 \alpha\langle u\rangle n_{0}\right)}=\infty, \quad\left|\lim _{c \rightarrow 1} \frac{3 \alpha / d}{2(c+2) n-3 \alpha\langle u\rangle}\right|<\frac{1}{d\langle u\rangle},
$$

we obtain

$$
\lim _{c \rightarrow 1} f(n, c)=\infty
$$

uniformly in $n$. Finally,

$$
\begin{aligned}
& \lim _{c \rightarrow \infty} \frac{6}{(c-1)\left(2(c+2) n_{0}^{2}-3 \alpha\langle u\rangle n_{0}\right)}=0, \\
& \lim _{c \rightarrow \infty} \frac{3 \alpha / d}{2(c+2) n-3 \alpha\langle u\rangle} \geq \frac{\alpha}{d n} \lim _{c \rightarrow \infty} \frac{3}{2(c+2)}=0
\end{aligned}
$$

because $n \mapsto \alpha /(d n)$ is bounded on $\left[s_{0}, n_{0}\right]$; hence, in view of (6.16),

$$
\liminf _{c \rightarrow \infty} f(n, c) \geq 0
$$

uniformly in $n$. Since the infimum of $f$ should be negative, we infer from the above results that

$$
\inf _{(n, c) \in\left[s_{0}, \infty\right) \times(1, \infty)} f(n, c)=\inf _{c \in(1, \infty)} f\left(s_{0}, c\right)=f\left(s_{0}, c_{+}\right),
$$

where $f\left(s_{0}, c_{+}\right)<0$ given by (6.14) provides an upper bound on the r.h.s. of (6.11) for the case $\iota=D N$.

Proof of Theorem 4.5, part (ii). In the Dirichlet case, we use the mollifier (6.12) with the fixed $n=s_{0}$ for the construction of the functions from $T^{D}$. We set for any $c_{1}, c_{2}>1$ and $\varepsilon \in \mathbb{R}$,

$$
\psi_{c_{1}, c_{2}, \varepsilon}(s, u):=\varphi_{c_{1}}\left(s ; s_{0}\right) \chi_{1}^{D}(u)+\varepsilon \varphi_{c_{2}}\left(s ; s_{0}\right) \chi_{2}^{D}(u)
$$

and $T^{D}:=\left\{\psi_{c_{1}, c_{2}, \varepsilon} \mid c_{1}, c_{2}>1, \varepsilon \in \mathbb{R}\right\}$. Easy explicit calculations give

$$
\begin{aligned}
Q_{1}^{D}\left[\psi_{c_{1}, c_{2}, \varepsilon}\right] & =\frac{\pi^{2}}{d}\left(h\left(c_{1}\right)+\frac{16}{3 \pi^{2}} \alpha \varepsilon+\varepsilon^{2}\left(2 g\left(c_{2}\right)+h\left(c_{2}\right)\right)\right), \\
\left\|\psi_{c_{1}, c_{2}, \varepsilon}\right\|^{2} & =\frac{2 d}{3}\left(g\left(c_{1}\right)+\frac{16}{3 \pi^{2}} \alpha \varepsilon+\varepsilon^{2} g\left(c_{2}\right)\right)
\end{aligned}
$$


where

$$
h(c):=\frac{2}{\pi^{2}} \frac{d}{s_{0}} \frac{1}{c-1}, \quad g(c):=\frac{s_{0}}{d}(c+2)-\frac{3}{4} \alpha .
$$

Thus, the quotient at the r.h.s. of (6.11) can be written as

$$
\tilde{f}\left(c_{1}, c_{2}, \varepsilon\right):=\frac{3 \pi^{2}}{2 d^{2}} \frac{h\left(c_{1}\right)+\frac{16}{3 \pi^{2}} \alpha \varepsilon+\varepsilon^{2}\left(2 g\left(c_{2}\right)+h\left(c_{2}\right)\right)}{g\left(c_{1}\right)+\frac{16}{3 \pi^{2}} \alpha \varepsilon+\varepsilon^{2} g\left(c_{2}\right)} .
$$

Clearly, $\tilde{f}$ is a continuous function of the three variables defined in the region $(1, \infty)^{2} \times \mathbb{R}$ (the denominator is positive since it is the squared norm of a nonzero function) and one could look for its infimum. However, from the technical point of view, it seems to be a rather complicated task and that is why we make first the following simplification.

We start by verifying that the infimum of $\tilde{f}$ is negative, i.e., $\psi_{c_{1}, c_{2}, \varepsilon}$ is an admissible trial function to estimate $\inf \left(H^{D}\right)-E_{1}^{D}<0$, cf Theorem 4.3. Obviously, $h(c)>0$ for any $c \in(1, \infty)$. Using the definition of $\alpha$, the assumption $\langle\mathbf{H}\rangle$ and obvious estimates, we check that the same holds true for $g$ :

$$
g(c)>3\left(\frac{s_{0}}{d}-\frac{\alpha}{4}\right)>3\left(\frac{s_{0}}{d}-\frac{1}{2} s_{0}\left\|k_{+}\right\|_{\infty}\right)>\frac{3}{2} \frac{s_{0}}{d} .
$$

Hence, the only term in the numerator of $\tilde{f}$ which can attain negative values is the term linear in $\varepsilon$. However, for any given $c_{2}>0$, there exists $\varepsilon \in \mathbb{R}$ of such a sign that $\alpha \varepsilon<0$ and with a sufficiently small absolute value so that the negative term linear in $\varepsilon$ dominates over the quadratic one. Then we can find $c_{1}$ large enough to make the numerator of the r.h.s. of (6.18) negative. Recalling that the denominator is positive, we can restrict ourselves to those values of the triple $\left(c_{1}, c_{2}, \varepsilon\right)$, for which $\tilde{f}\left(c_{1}, c_{2}, \varepsilon\right)<0$; let us denote $\mathcal{N}:=\left\{\left(c_{1}, c_{2}, \varepsilon\right) \in(1, \infty)^{2} \times \mathbb{R} \mid \tilde{f}\left(c_{1}, c_{2}, \varepsilon\right)<0\right\}$. Setting for any $\left(c_{1}, c_{2}, \varepsilon\right) \in \mathcal{N}$,

$$
f\left(c_{1}, c_{2}, \varepsilon\right):=\frac{3 \pi^{2}}{2 d^{2}} \frac{h\left(c_{1}\right)+\frac{16}{3 \pi^{2}} \alpha \varepsilon+\varepsilon^{2}\left(2 g\left(c_{2}\right)+h\left(c_{2}\right)\right)}{g\left(c_{1}\right)},
$$

we arrive easily at the inequality $\tilde{f}\left(c_{1}, c_{2}, \varepsilon\right) \leq f\left(c_{1}, c_{2}, \varepsilon\right)$, because the (positive) denominator in (6.18) is bounded from above by $g\left(c_{1}\right)$ due to the above considerations. Consequently,

$$
\inf \sigma\left(H^{D}\right)-E_{1}^{D} \leq \inf _{\left(c_{1}, c_{2}, \varepsilon\right) \in \mathcal{N}} f\left(c_{1}, c_{2}, \varepsilon\right) .
$$


Calculating the partial derivatives of $f$, it is straightforward to see that the system of equations $f_{, i}=0, i=1,2,3$, can be cast into the following form:

$$
\begin{aligned}
\frac{s_{0}}{d} A\left(c_{2}, \varepsilon\right)\left(c_{1}-1\right)^{2}+\frac{4}{\pi^{2}}\left(c_{1}-1\right)+\frac{6}{\pi^{2}} \frac{d}{s_{0}}\left(\frac{s_{0}}{d}-\frac{\alpha}{4}\right) & =0, \\
\left(c_{2}-1\right)^{2}-\left(\frac{d}{\pi s_{0}}\right)^{2} & =0, \\
\varepsilon+\frac{8 \alpha}{3 \pi^{2}} \frac{1}{h\left(c_{2}\right)+2 g\left(c_{2}\right)} & =0,
\end{aligned}
$$

respectively, where, for any $\left(c_{1}, c_{2}, \varepsilon\right) \in \mathcal{N}$,

$$
A\left(c_{2}, \varepsilon\right):=\frac{16}{3 \pi^{2}} \alpha \varepsilon+\varepsilon^{2}\left(2 g\left(c_{2}\right)+h\left(c_{2}\right)\right)<0 .
$$

From the second equation we can immediately express $c_{2}$; of course, we choose that root $c_{2+}$ which is greater than 1 . Substituting $c_{2+}$ to the third equation of our system, we obtain the root $\varepsilon_{0}$ (notice that really $\alpha \varepsilon_{0}<0$ ). Finally, putting $c_{2+}$ and $\varepsilon_{0}$ to the first equation, we choose that root $c_{1+}$ which is greater than 1. A tedious but straightforward calculation yields

$$
f\left(c_{1+}, c_{2+}, \varepsilon_{0}\right)=-\frac{3 \pi^{4}}{4 d^{2}} \frac{A\left(c_{2+}, \varepsilon_{0}\right)^{2}}{\left(1+\sqrt{1-\frac{3}{2} A\left(c_{2+}, \varepsilon_{0}\right) \pi^{2}\left(\frac{s_{0}}{d}-\frac{\alpha}{4}\right)}\right)^{2}}
$$

with

$$
A\left(c_{2+}, \varepsilon_{0}\right)=-\frac{32 \alpha^{2}}{9 \pi^{4}} \frac{1}{\frac{2}{\pi}+3\left(\frac{s_{0}}{d}-\frac{\alpha}{4}\right)} .
$$

(Recall that $\frac{s_{0}}{d}-\frac{\alpha}{4}>0$, so the square root in the first formula is well defined in $\mathbb{R}$.) Hence really $\left(c_{1+}, c_{2+}, \varepsilon_{0}\right) \in \mathcal{N}$. Moreover, one can check that the matrix of second derivatives of $f$ is in the point $\left(c_{1+}, c_{2+}, \varepsilon_{0}\right)$ diagonal with all positive elements, that is, the function $f$ reaches its local minimum in that point.

To see that it is the global minimum too, we study the behaviour of the limits of $f$ as $c_{i} \rightarrow 1, \infty, i \in\{1,2\}$ and $\varepsilon \rightarrow \pm \infty$. We restrict ourselves to that cases, where the limit is reached by negative values of $f$; the rest of the "boundary" of the set $\mathcal{N}$ consists of those triples $\left(\tilde{c}_{1}, \tilde{c}_{2}, \tilde{\varepsilon}\right)$, for which $f\left(\tilde{c}_{1}, \tilde{c}_{2}, \tilde{\varepsilon}\right)=0$, that is, $f\left(\tilde{c}_{1}, \tilde{c}_{2}, \tilde{\varepsilon}\right)>f\left(c_{1_{+}}, c_{2_{+}}, \varepsilon_{0}\right)$. Since (6.19) gives

$$
g(c)>\frac{3|\alpha|}{4}
$$

we obtain

$$
f\left(c_{1}, c_{2}, \varepsilon\right)>\frac{3 \pi^{2}}{2 d^{2}} \frac{\frac{16}{3 \pi^{2}} \alpha \varepsilon+\frac{3|\alpha|}{2} \varepsilon^{2}}{g\left(c_{1}\right)}
$$


and the condition $f\left(c_{1}, c_{2}, \varepsilon\right)<0$ yields

$$
|\varepsilon|<\frac{32}{9 \pi^{2}} .
$$

Hence we do not study the limits as $\varepsilon \rightarrow \pm \infty$ and we may assume in the following that $\varepsilon$ is bounded. Using (6.22) in the denominator of (6.20), neglecting $h\left(c_{1}\right)$ and minimizing the remaining polynomial in $\varepsilon$ in the numerator of (6.20), we arrive at the lower bound

$$
f\left(c_{1}, c_{2}, \varepsilon\right)>-\frac{128}{9 \pi^{2} d^{2}} \frac{|\alpha|}{h\left(c_{2}\right)+2 g\left(c_{2}\right)}
$$

for any $c_{2} \in(1, \infty)$. Thus

$$
\liminf _{c_{2} \rightarrow \infty} f\left(c_{1}, c_{2}, \varepsilon\right) \geq 0, \quad \liminf _{c_{2} \rightarrow 1} f\left(c_{1}, c_{2}, \varepsilon\right) \geq 0
$$

uniformly in $c_{1}$ and $\varepsilon$. Finally, using (6.23) we can see that

$$
f\left(c_{1}, c_{2}, \varepsilon\right)>\frac{3 \pi^{2}}{2 d^{2}} \frac{h\left(c_{1}\right)}{g\left(c_{1}\right)}-\frac{256|\alpha|}{9 \pi^{2} d^{2}} \frac{1}{g\left(c_{1}\right)}
$$

and therefore

$$
\liminf _{c_{1} \rightarrow \infty} f\left(c_{1}, c_{2}, \varepsilon\right) \geq 0, \quad \lim _{c_{1} \rightarrow 1} f\left(c_{1}, c_{2}, \varepsilon\right)=\infty
$$

uniformly in $c_{2}$ and $\varepsilon$. Summing up the considerations, we conclude that $f\left(c_{1_{+}}, c_{2_{+}}, \varepsilon_{0}\right)$ is the global minimum and the claim (ii) of Theorem 4.5 then follows from (6.21).

Remark 6.3 (Mildly curved strips). $\quad$ Let us compare our estimate (ii) of Theorem 4.5 with the exact ground-state eigenvalue asymptotics derived in [18, Thm. 4.1] for mildly curved Dirichlet strips by the Birman-Schwinger perturbation technique. We consider families of generating curves $\Gamma_{\beta}$ characterized by the curvature $k_{\beta}(s):=\beta k(s)$, where $k$ is a fixed curvature function and $\beta>0$ is a small parameter. Since $\alpha_{\beta}:=\int_{\mathbb{R}} k_{\beta}(s) d s=\beta \alpha$, we see that $\beta$ controls the total bending of the strip, too. The result of [18] can be written as

$$
\inf \left(H^{D}\right)=E_{1}^{D}-C(d, k)^{2} \beta^{4}+\mathcal{O}\left(\beta^{5}\right),
$$

where $C(d, k)$ is a positive constant depending only on the fixed width $d$ and (integrals of) $k$, while our estimate (ii) yields

$$
\inf \left(H^{D}\right) \leq E_{1}^{D}-C^{D}\left(s_{0}, d, 0\right)^{2} \alpha^{4} \beta^{4}+\mathcal{O}\left(\beta^{5}\right) .
$$


Hence we observe the same dependence of the leading terms on the perturbation parameter $\beta$. Let us quantitatively compare the actual gap-width asymptotic given by $C(d, k)^{2}$ with our estimate $C^{D}\left(s_{0}, d, 0\right)^{2} \alpha^{4}$. Since $C(d, k)$ has rather a complicated structure, we restrict ourselves to small values of the width $d$ when

$$
C(d, k)=\frac{1}{8}\|k\|_{L^{2}(\mathbb{R})}^{2}+\mathcal{O}\left(d^{2}\right) .
$$

We have $C^{D}\left(s_{0}, d, 0\right)=8 /\left(9 \sqrt{3} \pi^{2} s_{0}\right)+\mathcal{O}(d)$. Since $\alpha^{2} \leq 2 s_{0}\|k\|_{L^{2}(\mathbb{R})}^{2}$ by the Schwarz inequality, we see that

$$
\frac{C^{D}\left(s_{0}, 0,0\right) \alpha^{2}}{C(0, k)} \leq \frac{128}{9 \sqrt{3} \pi^{2}} \approx 0.83 .
$$

As for the mixed Dirichlet-Neumann case, our estimate (i) of Theorem 4.5 leads to

$$
\inf \left(H^{D N}\right) \leq E_{1}^{D N}-\frac{3 \alpha^{2}}{8 d^{2}} \beta^{2}+\mathcal{O}\left(\beta^{3}\right)
$$

and we observe that the leading term is proportional to the second power of $\beta$ now. In particular, it is much greater than the leading term in the identical mildly curved strip with the pure Dirichlet boundary condition. Unfortunately, no exact asymptotics are known for $\inf \sigma\left(H^{D N}\right)$, so we cannot perform any comparison in this case.

Remark 6.4 (Thin strips). Another natural perturbation parameter is the strip width $d$. Calculating the asymptotic expansions with respect to $d$ of the constants $C^{\iota}\left(s_{0}, d, \alpha\right)$ from our Theorem 4.5, we arrive at

$$
\begin{aligned}
E_{1}^{D N}-\inf \left(H^{D N}\right) & \geq-\frac{\alpha}{2 s_{0} d}+\mathcal{O}\left(d^{-\frac{1}{2}}\right), \\
E_{1}^{D}-\inf \left(H^{D}\right) & \geq \frac{2^{8} \alpha^{4}}{3^{5} \pi^{4} s_{0}^{2}\left(1+\sqrt{1+\left(\frac{4 \alpha}{3 \pi}\right)^{2}}\right)^{2}}+\mathcal{O}(d) .
\end{aligned}
$$

Again, we observe qualitatively different behaviour of our estimates with respect to the perturbation parameter.

In particular, the leading term in our lower estimate of the gap between the essential spectrum threshold and the lowest Dirichlet eigenvalue is independent of the strip width. This is in accordance with the perturbation expansion of the ground-state eigenvalue derived in [18, Thm. 5.1]:

$$
E_{1}^{D}-\inf \left(H^{D}\right)=-\lambda(k)+\mathcal{O}(d) .
$$


Here $\lambda(k)$ denotes the first (negative) eigenvalue of the one-dimensional Schrödinger operator $l:=-\Delta-\frac{1}{4} k^{2}$ on $L^{2}(\mathbb{R})$ with $\operatorname{Dom} l:=W^{2,2}(\mathbb{R})$, which is naturally associated with the problem and reflects the geometry of $\Gamma$ only. (We remark that, under our assumptions, the operator $l$ has always a negative eigenvalue, of [70, Thm. XIII.11].)

The leading term in the Dirichlet-Neumann estimate tends to $+\infty$ as $d \rightarrow 0$ (notice, however, that this fact does not conflict with anything because $E_{1}^{D N}=\mathcal{O}\left(d^{-2}\right)$ ). That is, we again observe the effect of stronger binding of the particle in the case when a Dirichlet boundary curve of the strip is replaced by the Neumann one. A similar asymptotic estimate can be also deduced directly from the crude bound (ii) of Proposition 6.1. Since no perturbation expansion with respect to $d$ for the lowest eigenvalue in the Dirichlet-Neumann case is known yet, we cannot compare our estimate with exact asymptotics.

\section{$\S 7 . \quad$ Conclusions}

Motivated by the theory of curved quantum waveguides, we were interested in spectral properties of the Laplace operator in a strip built over an infinite planar curve, see Figure 1, subject to three different types of boundary conditions (Dirichlet, Neumann or a combination of these ones, respectively). We localized the essential spectrum as a set under a very natural and weak condition about vanishing of curvature at infinity only, cf Theorem 4.1. We stress that no condition about the decay of derivatives of the curvature was required throughout this paper (the derivatives may not even exist because the reference curve is supposed to be $C^{2}$-smooth only). Then we were interested in the geometrically induced spectrum, i.e. the spectrum below the spectral threshold of the corresponding straight strip; we made a survey of known results and established new ones, $c f$ Theorems 4.2-4.4. Here the most important progress was achieved in the case of combined Dirichlet-Neumann boundary conditions where we generalized the only one known result of [17] and established two new sufficient conditions which guaranteed the existence of geometrically induced spectrum, $c f$ Theorem 4.4. We recall that the geometrically induced spectrum consists of discrete eigenvalues only whenever the above asymptotic behaviour of curvature holds true. Finally, we established two upper bounds to the spectral threshold in a situation when the geometrically induced spectrum is present, $c f$ Theorem 4.5. These estimates are new in the theory of curved quantum waveguides and their remarkable behaviour in the limit of mild curvature or small width of the strip was discussed, 
cf Remarks 6.3 and 6.4. Summing up briefly the main contribution of the paper, we gave answers to the two questions formulated in Section 2.

Let us now mention some directions in which the above mentioned results could be strengthened or extended.

In Theorem 4.1, we succeeded to localize the essential spectrum as a set, however, an open problem is to examine its nature. Here a particularly interesting question is whether the curved geometry may produce a singular continuous spectrum. In the Dirichlet case, this problem was analysed quite recently in [59] by means of the Mourre theory.

Theorem 4.3 concerning the existence of geometrically induced spectrum in Dirichlet strips is optimal in the sense that no better result can be achieved without violating the basic hypothesis $\langle\mathrm{H}\rangle$. One is of course tempted to ask which more general regions (than the curved asymptotically straight strips) still possess a non-trivial discrete spectrum. For instance, it is easy to see that the existence result does not change if the boundary of the strip is deformed locally and in such a way that the resulting deformed region lies in the exterior of the strip, cf [72], however, more complicated deformations of the boundary represent a difficult problem even in the straight case $[9,8]$. In this context, it is worth to recall that the existence of discrete spectrum in V-shaped waveguides was demonstrated in [43, 3, 11] (the computed bound-state energy has been verified experimentally in a flat electromagnetic waveguide in [10]).

The Neumann case is trivial from the point of view of the existence of discrete spectrum in asymptotically straight strips, $c f$ Theorem 4.2. As for the Dirichlet-Neumann strip, while our Theorem 4.4 covers various wide classes of geometries for which the geometrically induced spectrum exists, it does not represent an ultimate result. For instance, it remains to be clarified whether one can include also some thick strips with a positive total bending angle. Another open question concerning the strips with combined boundary condition is the study of the behaviour of eigenvalues in mildly curved, respectively thin, strips, cf Remarks 6.3 and 6.4.

The upper bounds on the spectral threshold we presented in Theorem 4.5 can be surely improved. First of all, one should include the situations when the total bending angle is equal to zero and/or the strip is curved globally.

As we have already mentioned in Introduction, the Dirichlet Laplacian in the curved strip represents a reasonable model for a quantum Hamiltonian of a particle restricted to move in a strip-like nanostructure. Assuming that the boundary is sufficiently regular, to impose the Dirichlet boundary conditions means to require the vanishing of wavefunctions, however, as pointed out 
in [50], this may be in general too restrictive and one should rather require the vanishing of the probability current only. The latter leads in our case to a general boundary condition of the type

$$
a_{0} \psi(\cdot, 0)+b_{0} \psi_{, 2}(\cdot, 0)=0, \quad a_{d} \psi(\cdot, d)+b_{d} \psi_{, 2}(\cdot, d)=0,
$$

where $\psi \in \mathcal{H}$ denotes the wavefunction and $\left(a_{0}, a_{d}\right),\left(b_{0}, b_{d}\right) \in \mathbb{R}^{2} \backslash\{(0,0)\}$. However, at least from the mathematical point of view, it would be interesting to examine the influence of the choice of particular boundary conditions on the spectral properties of the Hamiltonian. Finally, it would be also possible to let the coefficients $a_{0}, a_{d}, b_{0}, b_{d}$ depend on the longitudinal variable $s$.

Other obvious extensions are to consider the Laplacian in tubular neighbourhoods of non-compact submanifolds of general Riemannian manifolds. Here the spectral problem was studied only for Dirichlet tubes in $\mathbb{R}^{3}[52,18]$, Dirichlet layers in $\mathbb{R}^{3}[20,12]$ or more generally in $\mathbb{R}^{n}[64]$, and strips in twodimensional manifolds [58]; more general boundary conditions and other higherdimensional generalizations are still missing.

A long-standing open problem in the theory of quantum waveguides is the question whether the geometrically discrete spectrum in curved asymptotically straight Dirichlet strips will "survive" a strong homogeneous magnetic field. In this context, let us mention the very recent work [22] ( $c f$ also [5]), where it is shown actually that this is not the case for mildly curved strips if an appropriate compactly supported magnetic field is added.

\section{Acknowledgements}

One of the authors (D.K.) would like to thank V. Iftimie for pointing out to him the general characterization of essential spectrum (Lemma 5.1) which gives a better result than our original proof based on the Weyl criterion; discussions on this subject with I. Beltita are also acknowledged. We are very grateful to J. Dittrich, P. Duclos, P. Exner and P. Freitas for useful remarks and suggestions. The first author was partially supported by FCT/POCTI/FEDER, Portugal, and the AS CR project K1010104.

\section{References}

[1] Ashbaugh, M. S. and Exner, P., Lower bounds to bound state energies in bent tubes, Phys. Lett. A, 150 (1990), 183-186.

[2] Aslanyan, A., Parnovski, L. and Vassiliev, D., Complex resonances in acoustic waveguides, Quart. J. Mech. Appl. Math., 53 (2000), 429-447. 
[3] Avishai, Y., Bessis, D., Giraud, B. G. and Mantica, G., Quantum bound states in open geometries, Phys. Rev. B, 44 (1991), 8028-8034.

[4] Bentosela, F., Duclos, P. and Exner, P., Absolute continuity in periodic thin tubes and strongly coupled leaky wires, Lett. Math. Phys., 65 (2003), 75-82.

[5] Borisov, D., Ekholm, T., and Kovařík, H., Spectrum of the magnetic Schrödinger operator in a waveguide with combined boundary conditions, Ann. Henri Poincaré, (2001), 553-572.

[6] Borisov, D. and Exner, P., Exponential splitting of bound states in a waveguide with a pair of distant windows, J. Phys. A, 37 (2004), 3411-3428.

[7] Borisov, D., Exner, P., and Gadyl'shin, R., Geometric coupling thresholds in a twodimensional strip, J. Math. Phys., 43 (2002), 6265-6278.

[8] Borisov, D., Exner, P., Gadyl'shin, R. and Krejčiřík, D., Bound states in weakly deformed strips and layers, Ann. Henri Poincaré, 2 (2002), 553-572.

[9] Bulla, W., Gesztesy, F., Renger, W. and Simon, B., Weakly coupled bound states in quantum waveguides, Proc. Amer. Math. Soc., 125 (1997), 1487-1495.

[10] Carini, J. P., Londergan, J. T., Mullen, K. and Murdock, D. P., Bound states and resonances in waveguides and quantum wires, Phys. Rev. B, 46 (1992), 15538-15541.

[11] Multiple bound states in sharply bent waveguides, Phys. Rev. B, 48 (1993), 4503-4515.

[12] Carron, G., Exner, P. and Krejčiř́ík, D., Topologically nontrivial quantum layers, J. Math. Phys., 45 (2004), 774-784.

[13] Davies, E. B., Spectral theory and differential operators, Camb. Univ Press, Cambridge, 1995.

[14] Davies, E. B. and Parnovski, L., Trapped modes in acoustic waveguides, Quart. J. Mech. Appl. Math., 51 (1998), 477-492.

[15] Dermenjian, Y., Durand, M. and Iftimie, V., Spectral analysis of an acoustic multistratified perturbed cylinder, Comm. Partial Differential Equations, 23 (1998), 141-169.

[16] Dittrich, J. and Kříž, J., Bound states in straight quantum waveguides with combined boundary condition, J. Math. Phys., 43 (2002), 3892-3915.

[17] Curved planar quantum wires with Dirichlet and Neumann boundary conditions, J. Phys. A, 35 (2002), L269-275.

[18] Duclos, P. and Exner, P., Curvature-induced bound states in quantum waveguides in two and three dimensions, Rev. Math. Phys., 7 (1995), 73-102.

[19] Duclos, P., Exner, P. and Krejčiřík, D., Locally curved quantum layers, Ukrainian J. Phys., 45 (2000), 595-601.

[20] - Bound states in curved quantum layers, Comm. Math. Phys., 223 (2001), 13-28.

[21] Edmunds, D. E. and Evans, W. D., Spectral theory and differential operators, Oxford University Press, New York, 1987.

[22] Ekholm, T. and Kovař́k, H., Stability of the magnetic Schrödinger operator in a waveguide, Comm. Partial Differential Equations, to appear.

[23] Evans, D. V., Levitin, M. and Vassiliev, D., Existence theorems for trapped modes, J. Fluid Mech., 261 (1994), 21-31.

[24] Exner, P., Spectral properties of Schrödinger operators with a strongly attractive $\delta$ interaction supported by a surface, Contemp. Math., AMS, 339, Providence, R.I., 2003, Proceedings of the NSF Summer Research Conference (Mt. Holyoke 2002), pp. 25-36.

[25] Exner, P., Freitas, P. and Krejčiř́ík, D., A lower bound to the spectral threshold in curved tubes, R. Soc. Lond. Proc. Ser. A Math. Phys. Eng. Sci., 460 (2004), 3457-3467.

[26] Exner, P., Gawlista, R., Šeba, P. and Tater, M., Point interactions in a strip, Ann. Phys., 252 (1996), 133-179.

[27] Exner, P. and Ichinose, T., Geometrically induced spectrum in curved leaky wires, J. Phys. A, 34 (2001), 1439-1450.

[28] Exner, P., Joye, A. and Kovař́k, H., Edge currents in the absence of edges, Phys. Lett. A, 264 (1999), 124-130. 
[29] Exner, P., Joye, A. and Kovařík, H., Magnetic transport in a straight parabolic channel, J. Phys. A, 34 (2001), 9733-9752.

[30] Exner, P. and Kondej, S., Curvature-induced bound states for a $\delta$ interaction supported by a curve in $\mathbb{R}^{3}$, Ann. Henri Poincaré, 3 (2002), 967-981.

[31] $\longrightarrow$ Bound states due to a strong $\delta$ interaction supported by a curved surface, J. Phys. A, 36 (2003), 443-457.

[32] Strong-coupling asymptotic expansion for Schrödinger operators with a singular interaction supported by a curve in $\mathbb{R}^{3}$, Rev. Math. Phys., 16 (2004), 559-582.

[33] - Schrödinger operators with singular interactions: a model of tunneling resonances, J. Phys. A, 37 (2004), 8255-8277.

[34] - Leaky quantum wire and dots: a resonance model, math-ph/0307030.

[35] , Scattering by local deformations of a straight leaky wire, J. Phys. A, 38 (2005), 4865-4874.

[36] Exner, P. and Kovařík, H., Magnetic strip waveguides, J. Phys. A, 33 (2000), 3297-3311.

[37] Exner, P. and Krejčiř́k, D., Quantum waveguides with a lateral semitransparent barrier: Spectral and scattering properties, J. Phys. A, 32 (1999), 4475-4494.

[38] _ Bound states in mildly curved layers, J. Phys. A, 34 (2001), 5969-5985.

[39] Waveguides coupled through a semitransparent barrier: the weak-coupling behaviour, Rev. Math. Phys., 13 (2001), 307-334.

[40] Exner, P. and Němcová, K., Quantum mechanics of layers with a finite number of point perturbations, J. Math. Phys., 43 (2002), 1152-1184.

[41] , Leaky quantum graphs: approximations by point interaction Hamiltonians, J. Phys. A, 36 (2003), 10173-10193.

[42] Exner, P. and Šeba, P., Bound states in curved quantum waveguides, J. Math. Phys., 30 (1989), 2574-2580.

[43] Exner, P., Šeba, P. and Šťovíček, P., On existence of a bound state in an L-shaped waveguide, Czechoslovak J. Phys. B, 39 (1989), 1181-1191.

[44] Exner, P., Šeba, P., Tater, M. and Vaněk, D., Bound states and scattering in quantum waveguides coupled laterally through a boundary window, J. Math. Phys., 37 (1996), 4867-4887.

[45] Exner, P. and Vugalter, S. A., Asymptotic estimates for bound states in quantum waveguides coupled laterally through a narrow window, Ann. Inst. H. Poincaré, 65 (1996), 109-123.

[46] - Bound-state asymptotic estimates for window-coupled dirichlet strips and layers, J. Phys. A, 30 (1997), 7863-7878.

[47] B Bound states in a locally deformed waveguide: The critical case, Lett. Math. Phys., 39 (1997), 59-68.

[48] Exner, P. and Yoshitomi, K., Band gap of the Schrödinger operator with a strong $\delta$ interaction on a periodic curve, Ann. Henri Poncaré, 2 (2001), 1139-1158.

[49] Freitas, P. and Krejčiřík, D., A lower bound to the spectral threshold in curved strips with Dirichlet and Robin boundary conditions, submitted.

[50] Fülöp, T. and Tsutsui, I., A free particle on a circle with point interaction, Phys. Lett. A, 264 (2000), 366-374.

[51] Glazman, I. M., Direct methods of qualitative spectral analysis of singular differential operators, Israel Program for Scientific Translations, Jerusalem, 1965.

[52] Goldstone, J. and Jaffe, R. L., Bound states in twisting tubes, Phys. Rev. B, 45 (1992), 14100-14107.

[53] Hurt, N. E. Mathematical physics of quantum wires and devices, Kluwer, Dordrecht, 2000.

[54] Klaus, M., On the bound state of Schrödinger operators in one dimension, Ann. Phys., 108 (1977), 288-300.

[55] Kleespies, F. and Stollmann, P., Lifshitz asymptotics and localization for random quantum waveguides, Rev. Math. Phys., 12 (2000), 1345-1365.

[56] Klingenberg, W., A course in differential geometry, Springer-Verlag, New York, 1978. 
[57] Krejčiřík, D. Guides d'ondes quantiques bidimensionnels, Ph.D. thesis, Facultas Mathematica Physicaque, Universitas Carolina Pragensis; Faculté des Sciences et Techniques, Université de Toulon et du Var, September 2001, Supervisors: P. Duclos and P. Exner.

[58] , Quantum strips on surfaces, J. Geom. Phys., 45 (2003), 203-217.

[59] Krejčiŕík, D. and Tiedra de Aldecoa, R., The nature of the essential spectrum in curved quantum waveguides, J. Phys. A, 37 (2004), 5449-5466.

[60] Kreyszig, E., Differential geometry, University of Toronto Press, Toronto, 1959.

[61] Kříž, J., Spectral properties of planar quantum waveguides with combined boundary conditions, Ph.D. thesis, Facultas Mathematica Physicaque, Universitas Carolina Pragensis, April 2003, Supervisor: J. Dittrich.

[62] Kuchment, P. and Zeng, H., Convergence of spectra of mesoscopic systems collapsing onto a graph, J. Math. Anal. Appl., 258 (2001), 671-700.

[63] - Asymptotics of spectra of Neumann Laplacians in thin domains, Advances in differential equations and mathematical physics (Birmingham, AL, 2002), Contemp. Math., 327, Amer. Math. Soc., Providence, RI, 2003, pp. 199-213.

[64] Lin, Ch. and Lu, Z., Existence of bound states for layers built over hypersurfaces in $\mathbb{R}^{n+1}$, math.DG/0402252.

[65] Londergan, J. T., Carini, J. P. and Murdock, D. P., Binding and scattering in twodimensional systems, Lect. Note in Phys., m60, Springer, Berlin, 1999.

[66] Mekis, A., Fan, S. and Joannopoulos, J. D., Bound states in photonic crystal waveguides and waveguide bends, Phys. Rev. B, 58 (1998), 4809-4817.

[67] Newton, R. G., Bounds for the number of bound states for Schrödinger equation in one and two dimensions, J. Operator Theory, 10 (1983), 119-125.

[68] Olendski, O. and Mikhailovska, L., Localized-mode evolution in a curved planar waveguide with combined Dirichlet and Neumann boundary conditions, Phys. Rev. E, 67 (2003), art. 056625.

[69] Reed, M. and Simon, B., Methods of modern mathematical physics, I. Functional analysis, Academic Press, New York, 1972.

[70] Methods of modern mathematical physics, IV. Analysis of operators, Academic Press, New York, 1978.

[71] Rellich, F., Das Eigenwertproblem von $\Delta u+\lambda u=0$ in Halbröhren, Studies and Essays Presented to R. Courant on his 60th Birthday, January 8, 1948, Interscience Publishers, Inc., New York, 1948, pp. 329-344.

[72] Renger, W. and Bulla, W., Existence of bound states in quantum waveguides under weak conditions, Lett. Math. Phys., 35 (1995), 1-12.

[73] Seto, N., Bargmann's inequalities in spaces of arbitrary dimension, Publ. RIMS, Kyoto Univ., 9 (1974), 429-461.

[74] Shargorodsky, E. and Sobolev, A. V., Quasi-conformal mappings and periodic spectral problems in dimension two, J. Anal. Math., 91 (2003), 67-103.

[75] Weidmann, J., Linear operators in Hilbert spaces, Springer-Verlag, New York Inc., 1980.

[76] Yoshitomi, K., Band gap of the spectrum in periodically curved quantum waveguides, J. Differential Equations, 142 (1998), 123-166. 